\section{SANDIA REPORT}

SAND95-1794 • UC-712

Unlimited Release

Printed September 1995
TECENED

OCT 251995

\title{
Production of Lithium Positive lons from LiF Thin Films on the Anode in PBFA II
}

T. A. Green, R. W. Stinnett, R. A. Gerber, P. F. McKay, M. F. Lopez, R. A. Anderson,

T. D. Pointon, L. J. Lorence, Jr., J. A. Halbleib, A. B. Filuk, J. A. Panitz, P. R. Schwoebel

Prepared by

Sandia National Laboratories

Albuquerque, New Mexico 87185 and Livermore, California 94550

for the United States Department of Energy

under Contract DE-AC04-94AL85000

Approved for public release; distribution is unlimited. 
Issued by Sandia National Laboratories, operated for the United States Department of Energy by Sandia Corporation.

NOTICE: This report was prepared as an account of work sponsored by an agency of the United States Government. Neither the United States Government nor any agency thereof, nor any of their employees, nor any of their contractors, subcontractors, or their employees, makes any warranty, express or implied, or assumes any legal liability or responsibility for the accuracy, completeness, or usefulness of any information, apparatus, product, or process disclosed, or represents that its use would not infringe privately owned rights. Reference herein to any specific commercial product, process, or service by trade name, trademark, manufacturer, or otherwise, does not necessarily constitute or imply its endorsement, recommendation, or favoring by the United States Government, any agency thereof or any of their contractors or subcontractors. The views and opinions expressed herein do not necessarily state or reflect those of the United States Government, any agency thereof or any of their contractors.

Printed in the United States of America. This report has been reproduced directly from the best available copy.

Available to DOE and DOE contractors from

Office of Scientific and Technical Information

PO Box 62

Oak Ridge, TN 37831

Prices available from (615) 576-8401, FTS 626-8401

Available to the public from

National Technical Information Service

US Department of Commerce

5285 Port Royal Rd

Springfield, VA 22161

NTIS price codes

Printed copy: A03

Microfiche copy: A01 


\section{DISCLAIMER}

Portions of this document may be illegible in electronic image products. Images are produced from the best available original document. 


\title{
Production of Lithium Positive lons from LiF Thin Films on the Anode in PBFA II
}

\author{
T. A. Green, R. W. Stinnett, R. A. Gerber, P. F. McKay, M. F. Lopez \\ Beam Experiments Department \\ R. A. Anderson \\ Properties of Organic Materials Department \\ T. D. Pointon \\ Computational Electromagnetics and Plasma Physics Department
}

\author{
L. J. Lorence, Jr. and J. A. Halbleib \\ Simulation Technology Research Department \\ A. B. Filuk \\ Diagnostic Theory Department \\ Sandia National Laboratories \\ Albuquerque, NM 87185 \\ J. A. Panitz and P. R. Schwoebel \\ Department of Physics and Astronomy \\ University of New Mexico 87131
}

\begin{abstract}
The production of positive lithium ions using a lithium-fluoride-coated stainless steel anode in the particle beam fusion accelerator PBFA II is considered from both the experimental and theoretical points of view. It is concluded that the mechanism of $\mathrm{Li}^{+}$ion production is electric field desorption from the tenth-micron-scale crystallites which compose the columnar growth of the LiF thin film. The required electric field is estimated to be of the order of $5 \mathrm{MV} / \mathrm{cm}$. An essential feature of the mechanism is that the crystallites are rendered electronically conducting through electron-hole pair generation by $\mathrm{MeV}$ electron bombardment of the thin film during the operation of the diode. It is proposed that the ion emission mechanism is an electronic conductivity analogue to that discovered by Rollgen for lithium halide crystallites which were rendered ionically conducting by heating to several hundred degrees Celsius. Since an electric field desorption mechanism cannot operate if a surface flashover plasma has formed and reduced the anode electric field to low values, the possibility of flashover on the lithium fluoride coated anode of the PBFA II Li ${ }^{+}$ion source is studied theoretically. It is concluded with near certainty that flashover does not occur.
\end{abstract}




\section{Acknowledgment}

This work was supported jointly from May 1990 until Tom Green's retirement in January 1992 by D. R. Jennison's Solid State Theory Department and by R. W. Stinnett's Ion Sources Department. Tom Green wishes to thank George Samara, Dwight Jennison, Don Cook, and Regan Stinnett for making possible such an interesting application of alkali-halide science to the ion source for PBFA II. Thanks are also due to all the PBFA II workers for their friendliness and willingness to take time to bring a newcomer up to speed on this extremely complicated engineering project. Finally, Tom Green would like to thank Regan Stinnett for facilitating Tom's active participation with PBFA II experiments as a consultant until May 1993. Regan's intelligence, openmindedness, and positive attitude were an inspiration throughout. 


\section{Contents}

Acknowledgment ............................................................................................ ii

Contents ...................................................................................................

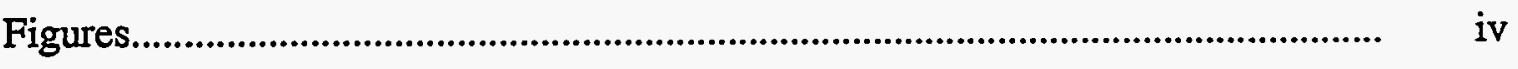

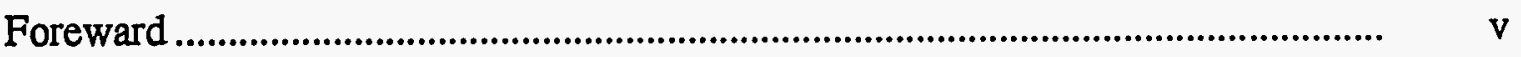

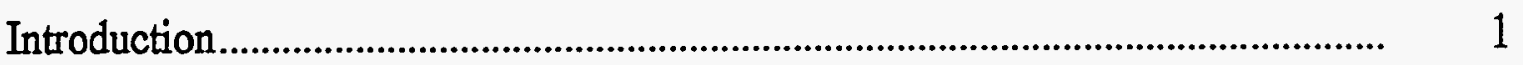

PBFA II experimental data ...................................................................................

General description of the accelerator and $\mathrm{LiF}$ ion source .................................. 2

PBFA II experiments using a LiF ion source...................................................... 4

Properties of the thin LiF film on PBFA II anode samples ..................................... 6

Field desorption of positive ions from ionic salt crystals ......................................... 10

Theory of bombardment-induced electronic conductivity in LiF thin-film coatings on metal anodes ........................................................................................................ 13

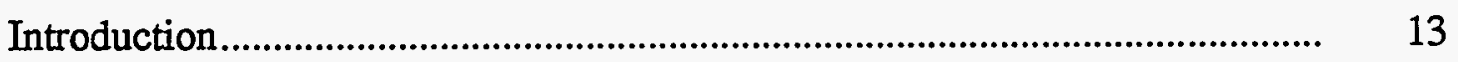

Electron and photon transport in the LiF-coated PBFA II anode......................... 14

QUICKSILVER particle in cell simulations of a PBFA II shot ........................... 16

Bombardment-induced heating, electronic conductivity, and surface charging in the presence of an external electric field .................................................................. 17

Recapitulation of the analogy between the electric field desorption in the LiF ion source and in the Rollgen experiments.................................................................. 23

Electron-bombardment-induced neutral ground-state fluorine atom production in

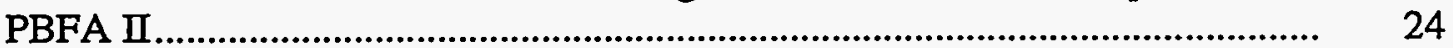

Flashover and dielectric breakdown on a LiF-coated anode in PBFA II................... 25

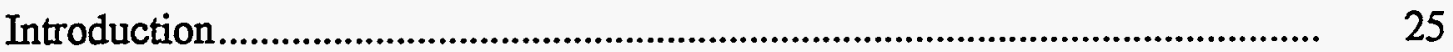

Theoretical discussion of flashover and dielectric breakdown on a $\mathrm{LiF}$ anode in

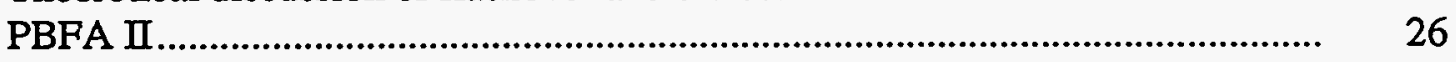

Principal fields, Larmor radius, effective frequency, and magnetic induction 26

Tangential electric fields .......................................................................... 27

Flashover across the LiF surface ................................................................ 28

Flashover between LiF columns ............................................................. $\quad 29$

Propagation of a surface discharge ............................................................. $\quad 30$

Stored energy available for Li desorption ..................................................... 30

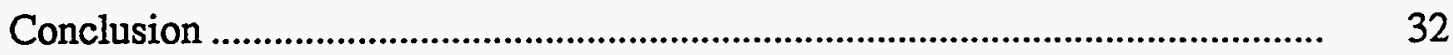

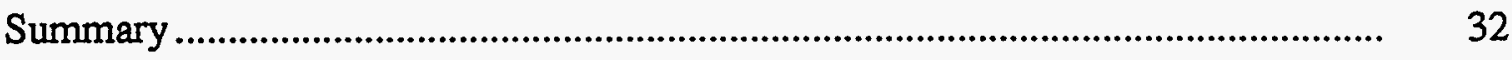

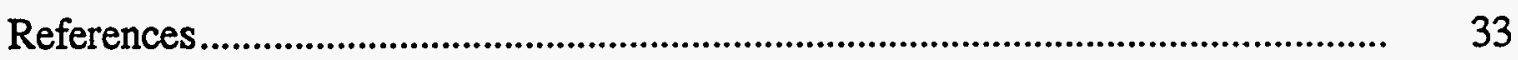




\section{Figures}

Figure 1. Schematic cross-section view of PBFA II center section ..................... 3

Figure 2. Typical waveforms for a LiF shot (\#6411) on PBFA II ..................... 5

Figure 3. High-resolution SEM images, taken at 45 degrees to normal, of $500 \mathrm{~nm} \mathrm{LiF}$ coating on rolled stainless steel anode sleeve sample......................... 7

Figure 4. SEM micrographs of various magnifications, looking straight down at LiFcoated anode....................................................................................... 8

Figure 5. TEM image of micron-thick LiF coating on a field-emission tip........ 9

Figure 6. XPS results for $3 \mathrm{LiF}$ samples showing percentage of detected species by atom versus depth into sample .......................................................... 10

Figure 7. Sublimation behavior of $\mathrm{KCl}$ crystals under field desorption condition 11

Figure 8. Intensity variation of $\mathrm{K}^{+}$signals during the field desorption process shown in Figure 7 .................................................................................... 12

Figure 9. Excitonic defects in an alkali-halide lattice ....................................... 12

Figure 10. Mono-energetic $\beta$ incident on LiF-coated stainless steel, showing Dose delivered into $5000 \AA$ of $\mathrm{LiF}$

Figure 11. Energy-angle distributions of electrons striking the anode from QUICKSILVER simulations

Figure 12. Calculated equipotentials in 2-dimensional case near the edge of a conductive layer 


\section{Foreward}

This SAND report is a revised version of a memo dated 3/21/93 that was distributed by Tom Green to members of the Sandia Light-Ion Inertial Confinement Fusion (ICF) Program. That memo is in turn a shortened version of an informal report to the Ion Sources Group in the ICF Program by Tom Green and Bob Anderson, entitled "Production of Lithium Positive Ions from Thin LiF Films Evaporated onto a Stainless Steel Anode". Due to the depth of the analysis, the expertise of the primary author in alkali-halides, and the relevance to on-going work in the ICF Program, a version was needed for external reference. With Tom Green's permission and final review, this document was completed by the addition of the experimental sections 2.1 and 2.2, and edited by Alex Filuk of Target \& Analysis Theory Department 1271 , for submission as a SAND report. Kevin Bieg graciously provided critical review of the final manuscript. 



\section{Production of Lithium Positive lons from LiF Thin Films on the Anode in PBFA II}

\section{Introduction}

Although the production of $\mathrm{Li}^{+}$ions from $\mathrm{LiF}$ thin films has been considered in a variety of contexts, the first evidence for the mechanism considered below was provided by experiments using a low-energy Physics International accelerator [1]-[3]. As discussed in References $1-3$, approximately $80 \%$ pure beams of $\mathrm{Li}^{+}$ions were generated from micronthickness $\mathrm{LiF}$ thin films on the anode of an extraction diode. The accelerator generated a 20-ns pulsed electric field of peak magnitude $1.8 \mathrm{MV} / \mathrm{cm}$ in a one-dimensional geometry. This field was insufficient to produce ions from a micron-thick $\mathrm{LiF}$ thin film on a polished anode substrate. However, when the local electric field was enhanced several times (or more) through the use of roughened stainless steel anode substrates, $\mathrm{Li}^{+}$average current densities of the order of $200 \mathrm{~A} / \mathrm{cm}^{2}$ were generated. The magnetic field applied to the diode to prevent electrons from crossing the gap from cathode to anode was about five times the critical magnetic field needed to insulate the gap, and the measured electron leakage current which bombarded the $\mathrm{LiF}$ thin film was about $15 \mathrm{~A} / \mathrm{cm}^{2}$. The relatively low values of the electric field compared to the expected value of about $100 \mathrm{MV} / \mathrm{cm}$ was puzzling, especially because direct electric field emission measurements confirmed that fields of the order of $100 \mathrm{MV} / \mathrm{cm}$ were indeed required to generate $\mathrm{Li}^{+}$ions from $\mathrm{LiF}$ thin films on a field emission tip at room temperature [4].

In $1988 \mathrm{LiF}$ coated stainless steel anodes began to be studied in PBFA II, with peak anode voltages of the order of $8 \mathrm{MV}$ and peak anode electric fields of the order of $10 \mathrm{MV}$ / $\mathrm{cm}$. Despite the extreme difficulty of measuring diode voltages, electric fields, electron and ion current densities, and particle species, it has gradually emerged that $\mathrm{Li}^{+}$current densities of the order of $1 \mathrm{kA} / \mathrm{cm}^{2}$ are produced on the best PBFA II shots and that there is a peak anode electric field during $\mathrm{Li}^{+}$ion production of the order of $10 \mathrm{MV} / \mathrm{cm}[5][6]$. The MeV electron leakage current striking the LiF thin film at near-grazing incidence has not been measured directly but is probably of the order of a few hundred $\mathrm{A} / \mathrm{cm}^{2}$.

The purpose of this paper is to report the most recent experimental and theoretical developments concerning the production of $\mathrm{Li}^{+}$ions from thin films of $\mathrm{LiF}$ on stainless steel anodes in PBFA II. The experimental section will present a summary of the data. These data benefit from recent improvements in PBFA II diagnostics. One new experimental finding of special interest here is that the micron-thick $\mathrm{LiF}$ thin film applied to the ion source anode consists of a crystalline columnar growth comprising some $10^{10}$ columns per $\mathrm{cm}^{2}$. Section 4 presents the new theory of the operation of the PI-110A and PBFA II LiF ion sources. It resolves the puzzle of $\mathrm{Li}^{+}$ion generation at surprisingly low electric fields by drawing an analogy to an existing positive ion source developed by Rollgen and his col- 
laborators [7]-[9]. They showed that individual $\mathrm{LiCl}$ and $\mathrm{LiI}$ crystallites grown on a tungsten wire - which also served to heat them to the point of adequate ionic conductivity would yield pure beams of $\mathrm{Li}^{+}$ions by electric field desorption in electric fields of several $\mathrm{MV} / \mathrm{cm}$. Similar positive ion emission at $\mathrm{MV} / \mathrm{cm}$ electric field strengths was found for other salts. Positive charging of the emitting surface was found to play an essential role in mediating electric field desorption at relatively low electric field strengths.

Two essential observations - one experimental, one theoretical - underlay the analogy drawn here. First is the observed high-areal-density columnar structure of the LiF anode thin film. It provides a way to obtain high $\mathrm{Li}^{+}$current densities from the currents emitted by the individual columns. Second is the theoretical determination that the $\mathrm{MeV}$ leakage electrons which strike the $\mathrm{LiF}$ thin film in a diode generate enough electron hole pairs to make the crystallites electronically conductive. The bombardment-induced electronic conductivity allows Rollgen's field desorption mechanism to operate on nanosecond time scales in the individual crystallites of the thin film.

The first goal of this paper is to define and justify the above analogy on theoretical grounds; more experimentation will be required for a rigorous proof. A second goal of this paper is to establish that the electric field desorption mechanism is consistent with the current information available from experiments on PBFA II. These experiments are discussed in Sections 2.1 and 2.2. The physical characteristics of the LiF thin film are discussed in Section 2.3. Rollgen's experiments are described in Section 3. In Section 4 the bombardment-induced conductivity of the LiF thin film is discussed. Then the analogy between the ionically-mediated field desorption mechanism and the operation of the ion sources is drawn. Finally the generation of neutral fluorine, which is a potential ion source contaminant, is discussed. Flashover of the LiF thin film in the PBFA II electric field is discussed in Section 5. This section is of particular importance because the plasma resulting from flashover would likely reduce the anode electric field to low values, preventing the electric field desorption mechanism from operating. A brief summary of the conclusions is provided in Section 6.

\section{PBFA II experimental data}

\subsection{General description of the accelerator and LiF ion source}

The PBFA II ion accelerator is a large pulsed power device that uses an applied-B ion diode to generate a $1 \mathrm{MA}, 10 \mathrm{MeV}, 20 \mathrm{~ns}$ pulse of $\mathrm{Li}^{+}$ions that can be focused onto an inertial confinement fusion target. The accelerator and its physical principals have been described previously [11], and detailed experiments [12] and theory [13] have been done to understand the physics governing the amount of beam power that can be generated. A very brief description of the ion diode will be given here to assist the reader unfamiliar with the device. Figure 1 shows the center section of PBFA II. The bi-conic power feeds are Magnetically-Insulated Transmission Lines (MTLs) that deliver a power pulse to the cylindrically-symmetric anode-cathode (AK) region. The diode impedance coupled to the MTLs results in the $\sim 10 \mathrm{MV}$ voltage across the AK gap when the power pulse arrives, so 


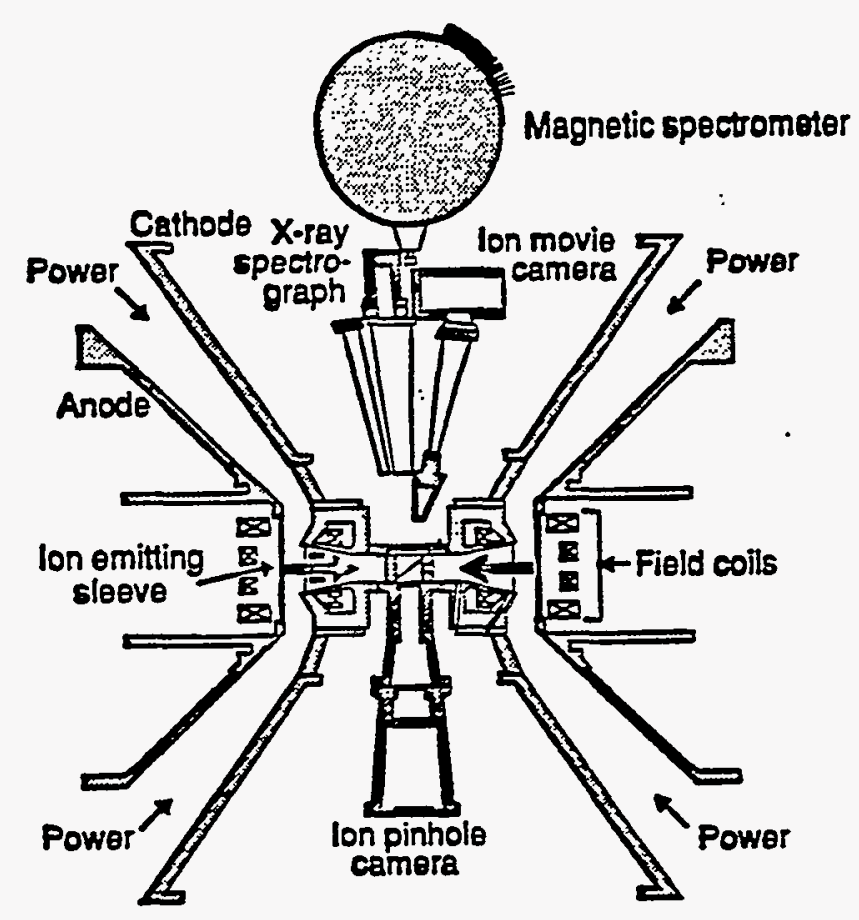

Figure 1. Schematic cross-section view of PBFA II center section, showing conical power feeds and some of the ion beam diagnostics that view the beam focus on-axis. Ion beam is accelerated radially inward from LiF-coated ion emitting sleeve to centerline..

that ions are accelerated from the anode across the $\sim 2 \mathrm{~cm}$ gap radially inwards to the target. Anode and cathode field coils create an applied magnetic field to prevent electrons from crossing the AK gap. The effectiveness of this electron insulation depends on the shape of the magnetic flux surfaces, and the degree of azimuthal symmetry of the charge distributions in the AK gap; azimuthal asymmetries due to small hardware misalignments or defects, electron emission spots, or collective effects (instabilities) can all give rise to electron leakage current to the anode. The electrons in the AK gap EXB drift relativistically in the azimuthal direction. The anode ion source is the inside surface of the $\sim 15 \mathrm{~cm}$ radius cylindrical sleeve in Figure 1. This surface may be concave to assist in focusing the radially-converging ion beam on the target at the machine center. The inner surface of the stainless steel anode sleeve is cleaned with solvents and baked for several hours at $\sim 200 \mathrm{C}$ prior to evaporation of typically $0.5 \mu \mathrm{m}$ of LiF. Coatings as thin as $600 \AA$ have been used. The surface roughness of the stainless has been varied as described below.

An extensive set of diagnostics has been developed to determine the conditions in the AK gap and the properties of the ion beam. These have also been described in detail previously [14], and include Faraday cups, B-dot current monitors, nuclear activation, ion pinhole multi-frame camera, Thomson parabola, AK gap visible spectroscopy, and an ion magnetic spectrometer. The Faraday cups have several different filters and hole sizes to discriminate various types of ions and to look for cup hole closure effects. A limitation of these cups is that they although they can readily be used to distinguish proton from nonproton ions, they cannot easily be used to discriminate lithium ions from other non-protonic 
species such as carbon ions. However, the on-axis Rutherford-scattering magnetic spectrometer is a species-discriminating diagnostic that can be used to obtain both the lithium ion voltage and current at the beam focus.

The AK gap spectroscopy uses fiber optics to view optical emissions from the region in front of the $\mathrm{LiF}$ anode. A key observation relevant to the $\mathrm{LiF}$ source is the measurement of electric field in the AK gap from the Stark shift of line emission [12]. The line-of-sight is a long pencil-shaped cylinder of about $2 \mathrm{~mm}$ diameter running parallel to the machine axis of symmetry and the anode surface. By partially overlapping this line-of-sight with the anode, the Stark shift of light gives an average electric field within $1 \mathrm{~mm}$ of the anode surface. A lower bound for the electric field at the anode can be obtained from this by allowing for the effect of ion space charge within this $1 \mathrm{~mm}$ distance to the anode. The amount of ion space charge can be determined self-consistently using the average electric field and the ion beam current measured with a Faraday cup.

\subsection{PBFA II experiments using a LiF ion source}

Observations relevant to the $\mathrm{LiF}$ ion source are described here in the sequence of events at the ion diode during a typical shot. Some information on the electron behavior is obtained from the detailed QUICKSILVER 3-dimensional electromagnetic particle-in-cell simulations [15]-[17] since it is not measured directly on PBFA II.

As the power pulse arrives at the diode and a several MV potential difference appears across the anode and cathode, electrons are drawn from the highest-field regions of the cathode tips and the power feed near the diode. These electrons move along flux surfaces into the AK gap region; their charge distribution modifies the vacuum electric field across the gap. The intense crossed electric and magnetic fields create a strong electron EXB drift azimuthally in the AK gap, and the resulting diamagnetic current begins to compress magnetic flux and the virtual cathode position toward the anode. The sheared electron flow at the two free surfaces of the drifting population gives rise to a diocotron instability, which in turn can break the azimuthal symmetry and allow a fraction of the electrons to reach the anode surface. These multi-MeV electrons enter the anode surface at a grazing angle and deposit their energy. The voltage across the diode reaches over $9 \mathrm{MV}$, high enough that ion emission should be easily detected if the $\mathrm{LiF}$ were a space-charge-limited emitter. Yet all of this happens prior to any measurable ion emission from the $\mathrm{LiF}$ anode, at $\mathrm{t}<39 \mathrm{~ns}$ as shown in the waveforms of Figure 2 for a typical shot (\#6411).

The first ions measured in the Faraday cups and magnetic spectrometer appear to be only $\mathrm{Li}^{+}$, although the Thomson Parabola shows a carbon ion signal at similar energy that may be due to $\mathrm{C}^{+2}$ appearing later in the pulse when the diode voltage has fallen to about half its original value. Crude species discrimination is determined in the Faraday cups by the ion charge stripping fraction from a comparison of a cup signal with a stripping filter to that without a filter. One can easily determine ion species in the magnetic spectrometer using predicted focus position and ion time-of-flight. As noted above, in earlier LiF experiments at lower voltages and AK gap electric fields increasing the LiF substrate roughness from 10 to $30 \mu \mathrm{m}$ had no effect on the $\mathrm{Li}^{+}$turn-on time but did increase the $\mathrm{Li}^{+}$beam cur- 


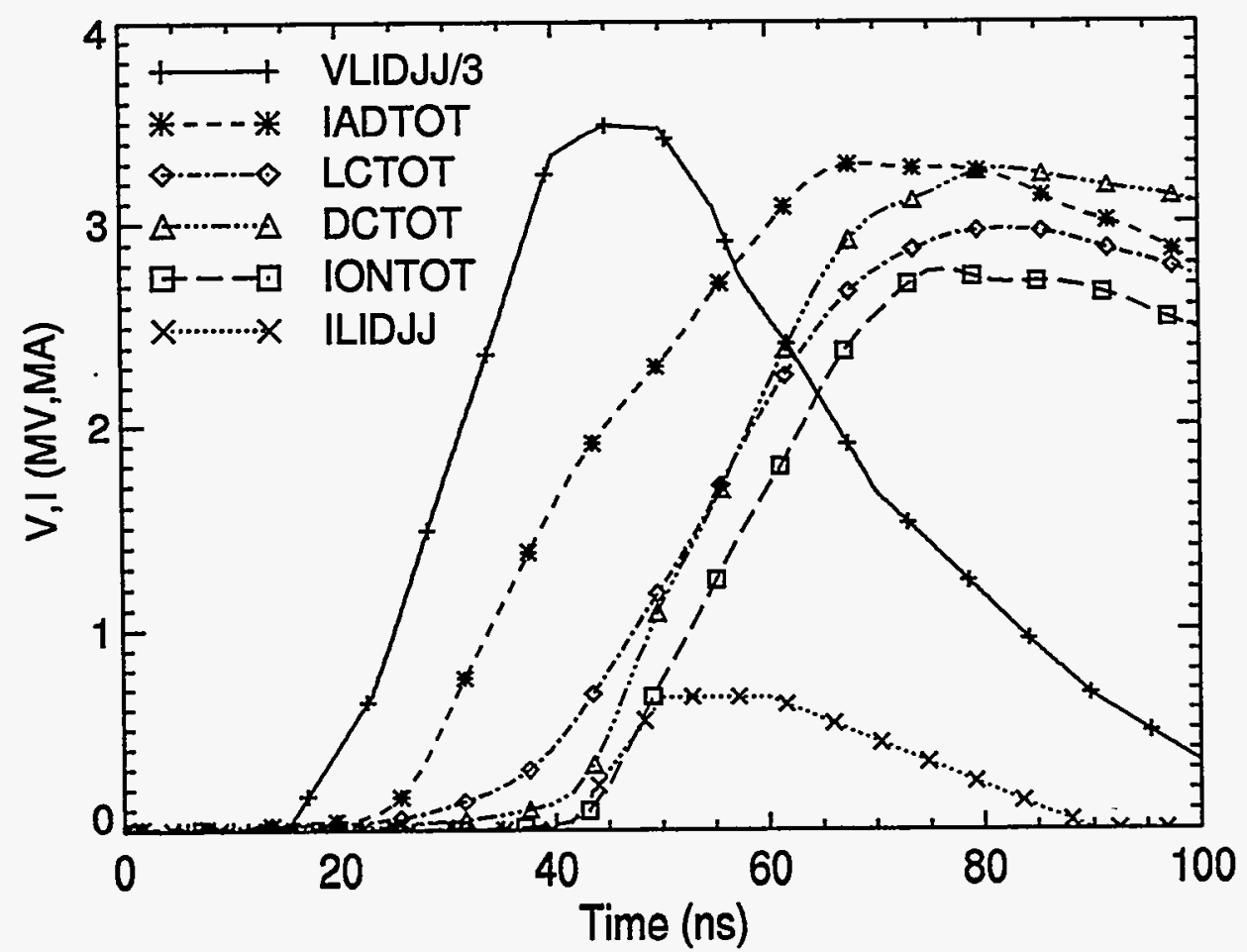

Figure 2. Typical waveforms for a $\mathrm{LiF}$ shot (\#6411) on PBFA II. VLIDJJ/3 is $1 / 3$ the $\mathrm{Li}^{+}$ ion energy and ILIDJJ is the $\mathrm{Li}^{+}$ion current as measured on-axis with the Magnetic Spectrometer (note VLIDJJ/3 prior to $t=40 \mathrm{~ns}$ is an estimate based on current measurements. IADTOT is the anode-side current measured using B-dots just outside the LiF-coated anode region. LCTOT, DCTOT, and IONTOT are cathodeside B-dot currents measured in the near-diode MITL feed, just outside the cathode tips opposite IADTOT, and inside the cathode tips, respectively.

rent. In more recent PBFA II experiments at $\sim 10 \mathrm{MV} / \mathrm{cm}$ fields the stainless steel substrate was polished to reduce the surface roughness from $\sim 1 \mu \mathrm{m}$ down to $0.03 \mu \mathrm{m}$, and no significant changes in the $\mathrm{Li}^{+}$turn-on time or the beam current were seen. These observations indicate that the $\mathrm{LiF}$ surface electric field is important for ion emission, but the source turnon requires more than simply applying a field larger than some threshold.

No spectral emission is seen at the anode in the gap spectroscopy prior to $\mathrm{Li}^{+}$ion turnon. The diode voltage is typically 9-10 MV when the $\mathrm{LiF}$ turns on. Within a few ns of the turn-on Li I 2s-2p (6708 $\AA$ ) emission is seen within $1 \mathrm{~mm}$ of the anode, with a Stark shift indicating an average electric field strength of typically $8-10 \mathrm{MV} / \mathrm{cm}$ (this neutral $\mathrm{Li}$ has several $\mathrm{keV}$ energy and is believed to be created by $\sim 1 / 20$ of the $\mathrm{Li}^{+}$beam ions undergoing charge-exchange with desorbed impurities near the anode surface). This electric field is also approximately the field at the anode surface at ion turn-on since the ion space-charge effect is very small when the ion beam current is still low. The large anode field persists even as the ion current rises, although by about $15 \mathrm{~ns}$ into the $\mathrm{Li}^{+}$emission the ion current is large enough that the ion space-charge could reduce the field at the anode to zero while maintaining the observed non-zero field at the near-anode spectroscopic line-of-sight. However, the ion space-charge needed to accomplish this would require the complete absence of electrons within $1 \mathrm{~mm}$ of the anode, contrary to the expected evolution of the diode electron distribution toward the anode later in the power pulse (so-called "saturation" 
of the electron sheath). Conservatively, we can say that for much of the period when $\mathrm{Li}^{+}$ emission occurs the anode electric field is non-zero, and we cannot tell if the anode field is zero for the rest of this period. Normally, during the $\mathrm{Li}^{+}$ion current rise no significant amount of beam impurity ions are seen in the beam diagnostics.

The $\mathrm{Li}^{+}$current density rises to $0.5-1 \mathrm{kA} / \mathrm{cm}^{2}$, then flattens and falls off while the total beam current continues to rise. Refer to signals IONTOT and ILIDJJ for $t>50 \mathrm{~ns}$ in Figure 2. Note that on this shot the LiF-coated anode area was about $940 \mathrm{~cm}^{2}$. Prior to this time little or no ion spectral lines are seen at the anode, either from $\mathrm{Li}^{+}$or from other anode impurities such as $\mathrm{C}^{+}-\mathrm{C}^{+3}$ that are seen later. On other shots with two magnetic spectrometers it was seen that the energy of the $\mathrm{Li}^{+}$beam in the on-axis magnetic spectrometer agreed well with the diode voltage as measured with another magnetic spectrometer in the near-diode MITL feed region, providing evidence that the $\mathrm{Li}^{+}$ions originate at or very near the anode surface. The total energy in the $\mathrm{Li}^{+}$beam as measured with nuclear activation and the magnetic spectrometer agree well, and confirm that the $\mathrm{Li}^{+}$current does fall off rather than simply miss the on-axis spectrometer scattering foil. This clamping and drop of the $\mathrm{Li}^{+}$current is the subject of current work on PBFA II, and will not be dealt with here as we are addressing the $\mathrm{LiF} \mathrm{Li}^{+}$ion source mechanism. Thus we are interested in explaining the observation of apparently-pure $\mathrm{Li}^{+}$ion emission for the first 10-15 ns of the beam.

\subsection{Properties of the thin LiF film on PBFA II anode samples}

As a step toward investigating the possible contamination of $\mathrm{LiF}$ in PBFA II ion sources, and in order to better see what the evaporated LiF thin film on a PBFA II anode looks like at high magnification, two types of experiments, scanning electron microscope (SEM) and x-ray photoelectron spectroscopy (XPS), were carried out. All the samples were prepared by evaporating LiF onto PBFA II anode sleeve samples during regular anode coating runs in which anodes for future shots were being prepared. The samples were stored in an Ar-filled glove box until they were used.

Figure 3 shows a typical high resolution SEM image, taken at a 45 degree angle to the surface normal, of a $500 \mathrm{~nm}$ LiF coating on a rolled stainless steel anode sleeve sample. The micron-scale cracks and bumps in the coating reflect the surface roughness of the stainless steel substrate. The columnar growth is evident and the density of columns is about $10^{10} \mathrm{~cm}^{-2}$. Figure 4 shows SEM micrographs looking straight down on the anode. Micronthick coatings from the PBFA II coating laboratory always exhibit this type of columnar growth, regardless of whether the metal substrate is rolled stainless steel, is roughened by bead blasting, or has grooves machined into it. High-resolution SEM images of the PI-110A anodes were not taken. However the columnar growth shows up clearly in the lower-resolution SEM image for a spray-acid-etched stainless steel anode. As shown in the transmission electron microscope (TEM) image in Figure 5, the columnar growth also always occurs when micron-thick LiF coatings are produced on field emission tips [18].

The same samples used for SEM studies allowed XPS studies of the surface atomic composition of anode samples under various conditions to be carried out at Sandia. XPS depends on inner shell photoionization by $\mathrm{Mg} \mathrm{K}_{\alpha} \mathrm{x}$-rays, and does not detect hydrogen. 

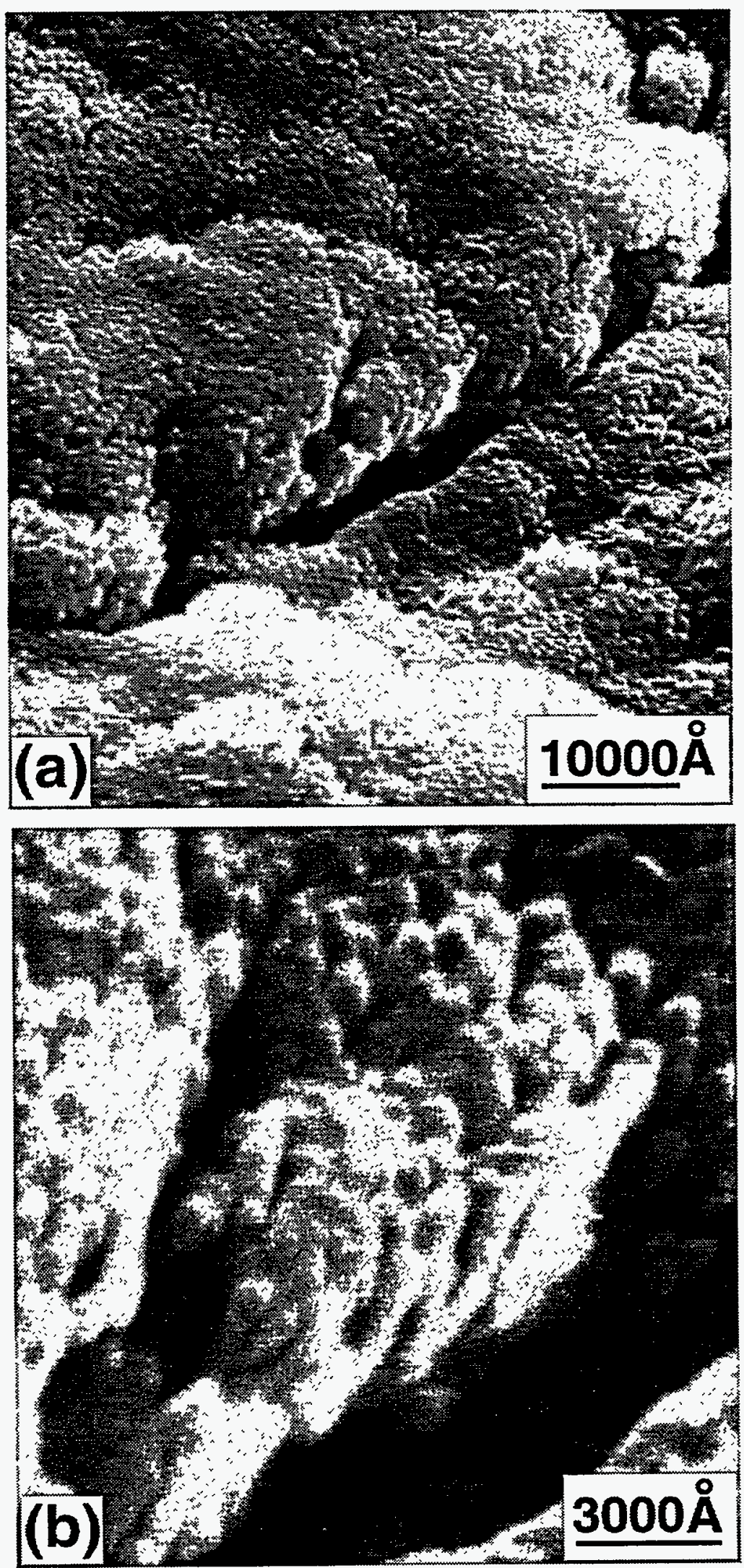

Figure 3. High-resolution SEM images, taken at 45 degrees to normal, of $500 \mathrm{~nm} \mathrm{LiF}$ coating on rolled stainless steel anode sleeve sample. Lower image is enlargement of central region of upper image. 
Production of Lithium Positive Ions from LiF Thin Films on the Anode in PBFA II
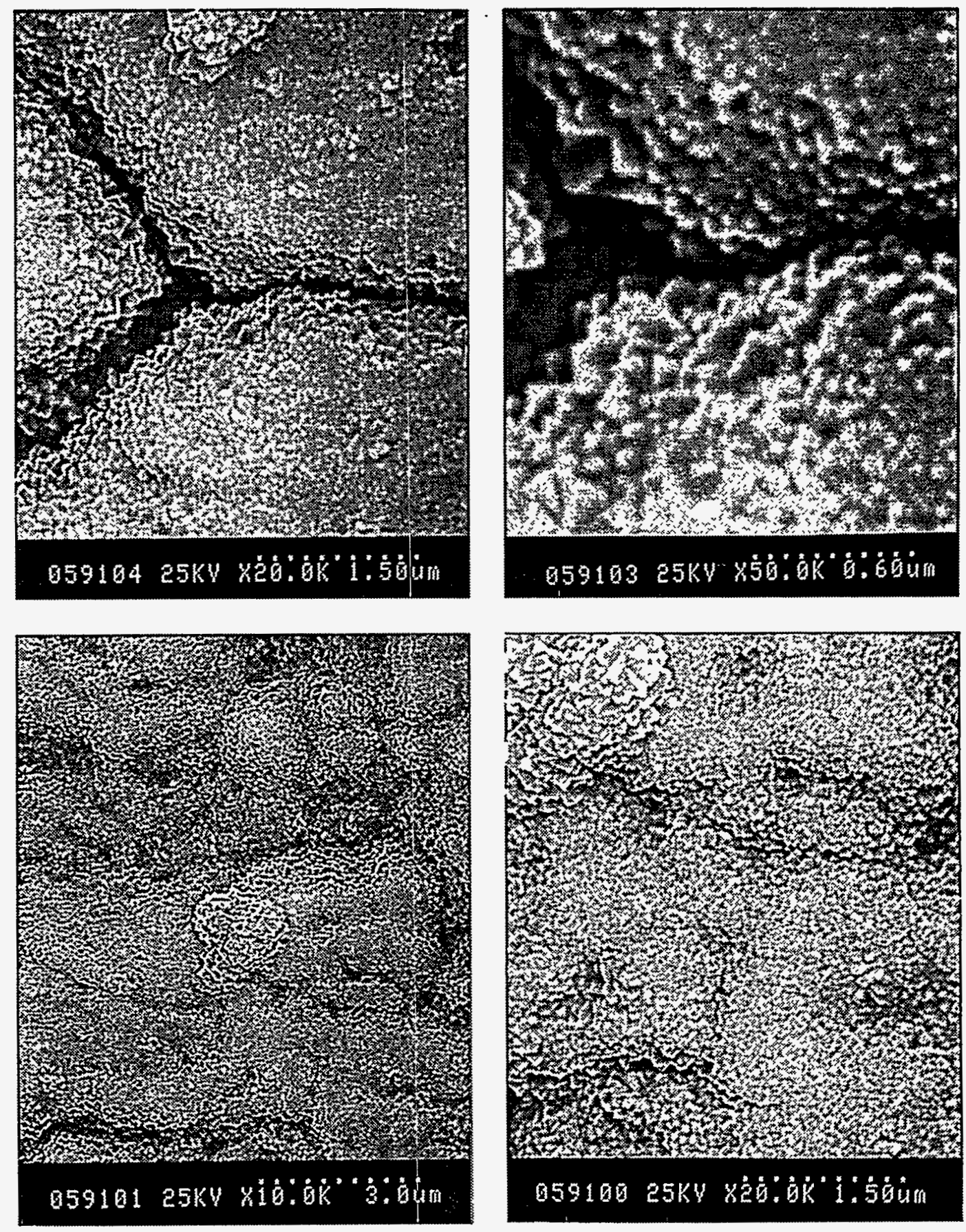

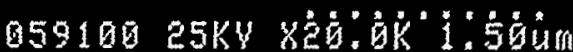

Figure 4. SEM micrographs of various magnifications, looking straight down at LiF-coated anode. 


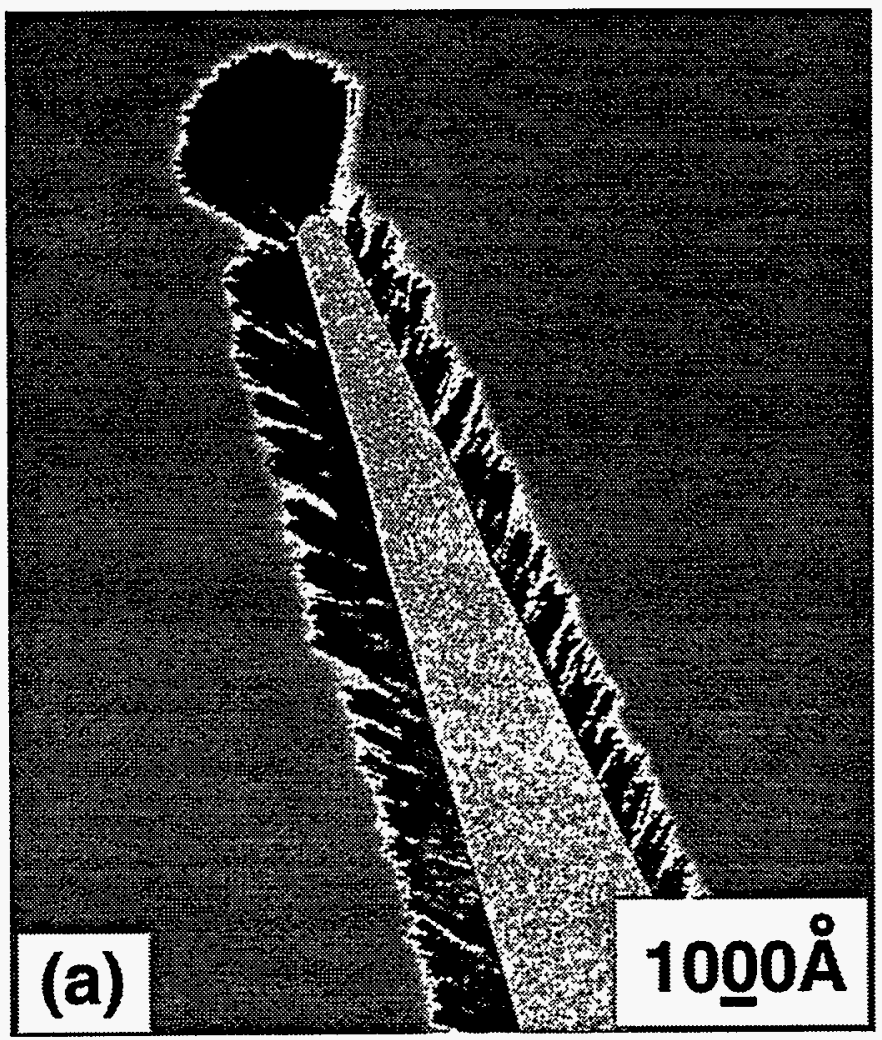

Figure 5. TEM image of micron-thick LiF coating on a field-emission tip. Note columnar structure of coating.

Because only un-scattered photoelectrons and Auger electrons are used in the analysis, the method samples a depth of a few atomic layers. Deeper regions are studied by progressively removing previously-studied layers with ion bombardment. One thus obtains the atomic composition as a function of depth below the surface. Figure 6 exhibits the results of three studies of $5000 \AA$ coated anode samples. The plots show the atomic percents determined for $\mathrm{Li}(1 \mathrm{~s}), \mathrm{F}(2 \mathrm{~s}), \mathrm{C}(1 \mathrm{~s})$, and $\mathrm{O}(1 \mathrm{~s})$ as a function of the depth of material sputtered off the surface. $\operatorname{Si}(2 \mathrm{p})$ and $\operatorname{Ar}(2 \mathrm{p})$ were detected in amounts $<1 \%$ and are not shown. Sample gl03 sat in the air in the XPS lab for about a week after being coated and shows increased carbon compared to sample g303 which was studied within an hour of being coated. Sample g303 is quite clean. By contrast sample g302 has one $\mathrm{C}$ atom for every two $\mathrm{Li}$ atoms in the first few atomic layers ( $21 \% \mathrm{C}$ and $39 \% \mathrm{Li}$ at the surface). This sample was placed close to the anode location in the center section of PBFA II for several hours while the anode and cathode elements were assembled for a LiF shot. The sample was removed for immediate analysis just before the machine was closed up prior to pumpdown. The experiments show that the evaporative coating process produces an adequately clean LiF anode which is presumably contaminated with carbon at the monolayer coverage level while it is being placed into the machine. Since XPS does not reveal hydrogen, and since the optical spectroscopy during a PBFA II shot reveals the presence of significant hydrogen as well as carbon, it is likely that the $\mathrm{LiF}$ is also contaminated with hydrogen. Note that the oxygen contamination is always small. This is consistent with evidence from the literature that indicates that, at room temperature, water does not stick to $\mathrm{LiF}$ in significant amounts [19][20]. 

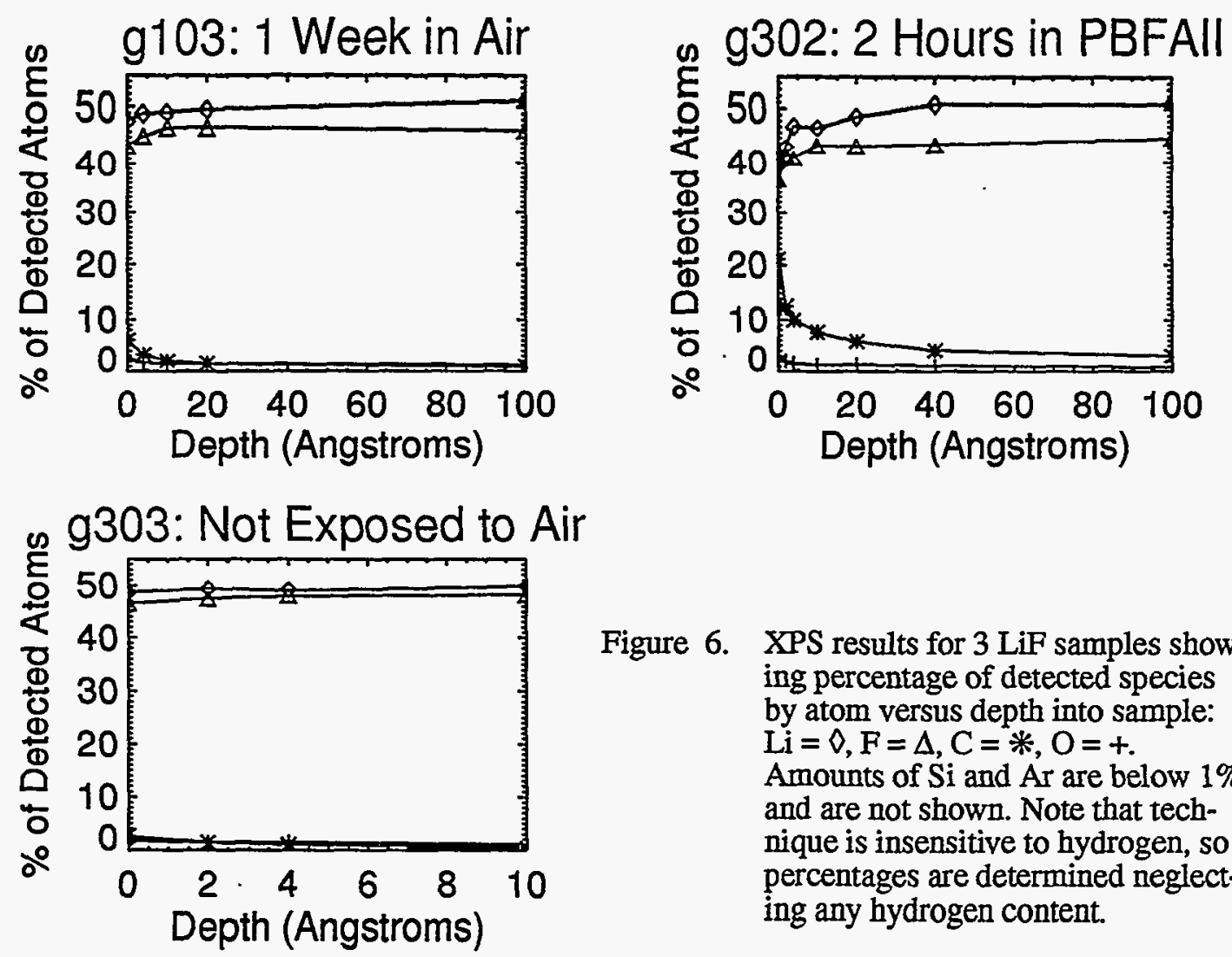

Figure 6. XPS results for $3 \mathrm{LiF}$ samples showing percentage of detected species by atom versus depth into sample: $\mathrm{Li}=\emptyset, \mathrm{F}=\Delta, \mathrm{C}=*$, $\mathrm{O}=+$. Amounts of Si and Ar are below $1 \%$ and are not shown. Note that technique is insensitive to hydrogen, so percentages are determined neglecting any hydrogen content.

\section{Field desorption of positive ions from ionic salt crystals}

As stated in the Introduction, electric field desorption of $\mathrm{Li}^{+}$ions from $\mathrm{LiCl}$ and $\mathrm{LiI}$ has been used for the production of pure, low-current, steady-state $\mathrm{Li}^{+}$ion beams for mass spectrometry by Rollgen and collaborators [7]-[9]. The technique consists of growing crystallites on a tungsten wire which supports them and serves to heat them to about $500^{\circ} \mathrm{C}$ in the presence of an average electric field of several $\mathrm{MV} / \mathrm{cm}$, as determined by the wire radius. A photograph of the ion source apparatus showing a $10 \mu \mathrm{m}$ diameter wire and a similar-sized crystallite of $\mathrm{KCl}$ is shown in Figure 1 of Reference 7 and is reproduced here as Figure 7. The Figure shows an evaporating $\mathrm{KCl}$ crystal at times a-h which correspond to the ion currents plotted in Figure 2 of Reference 7, reproduced here as Figure 8. The signal drops about an order of magnitude when the crystal disappears. The ions pass through the device's cathode to a mass spectrometer for analysis. Reference 8 exhibits the mass spectrometer signal for a $\mathrm{LiCl}$ source. In Reference 9 the authors ascribe the field desorption to the process of thermally activated charging of the surface by excess $\mathrm{Li}^{+}$ions, accompanied by ionic conduction in which an excess of negative halogen ions accumulates at the tungsten wire interface. Here they may undergo chemical reactions as in electrolysis [9]. The heating of the salts produces cation and anion vacancies - missing lattice ions in the cubic lattice of alternating positive and negative ions. These vacancies and the bombardmentinduced excitonic defects which are essential to the electronic analogy which will be drawn 

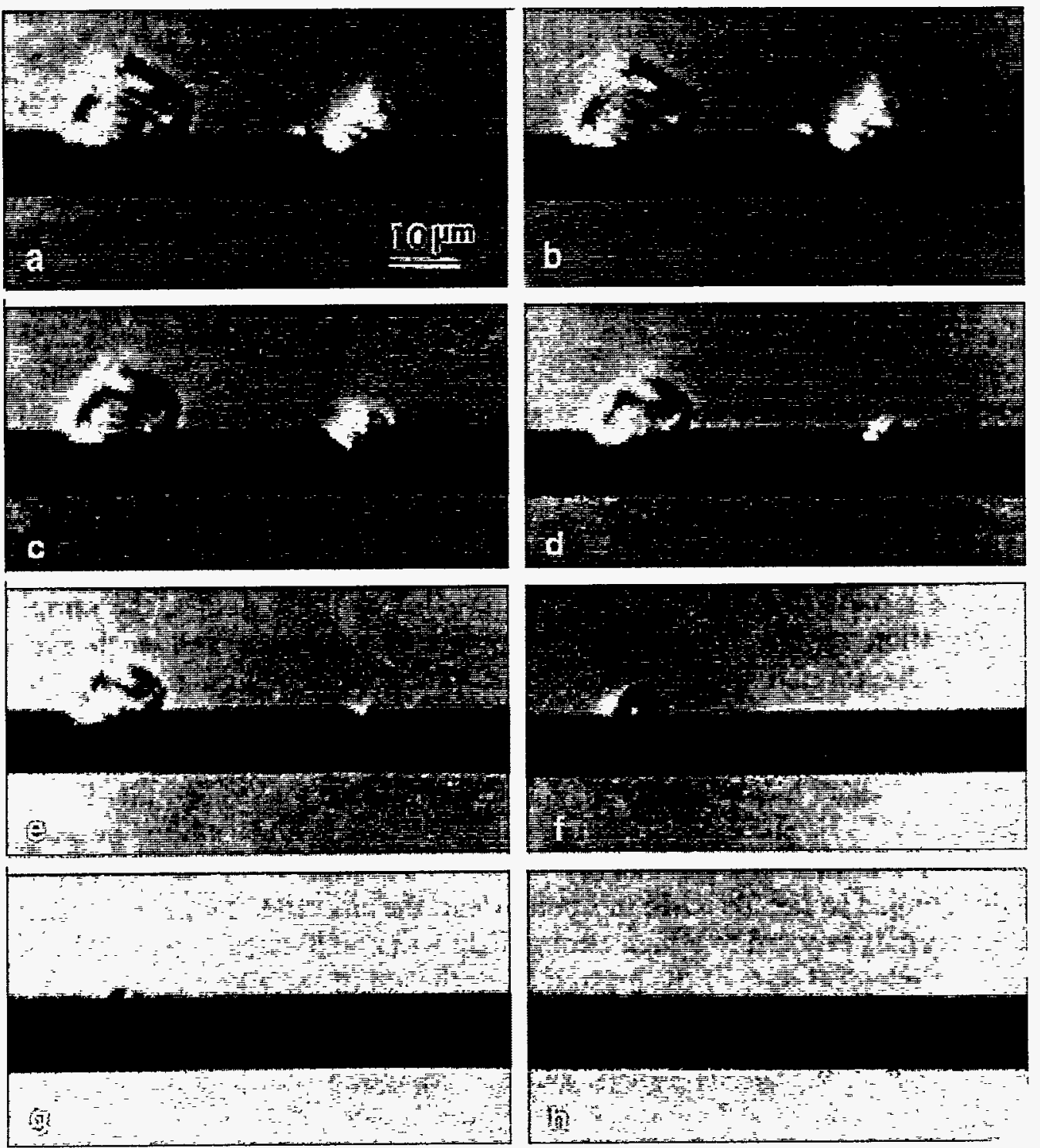

Figure 7. Sublimation behavior of $\mathrm{KCl}$ crystals under field desorption condition: $\mathrm{b}$ ) before heating, b)-h) morphological change of the crystals progressively observed at $\sim 500$ degrees $C$. The time from $f$ ) to $h$ ) is about 1 minute. Figure from Reference 7, reproduced with permission.

in Section 4 can be understood from Figure 9 [10]. For the present the reader should ignore the excitonic defects and consider only the perfect lattice of alternating positive and negative ions. In the perfect lattice no ionic conduction is possible because there is no room for translational motion. At temperatures of several hundred degrees $\mathrm{C}$, metal ion (cation) and halogen ion (anion) vacancies will be distributed around the lattice providing spaces into which neighboring ions can move. A cation vacancy behaves like a negative charge; an anion vacancy behaves like a positive charge. This allows a net transport of charge. A concentration of anion vacancies at the surface amounts to a local excess of positive ions. The authors conclude that the accumulation of positive charge at the emitting surface lowers the 
Production of Lithium Positive Ions from LiF Thin Films on the Anode in PBFA II

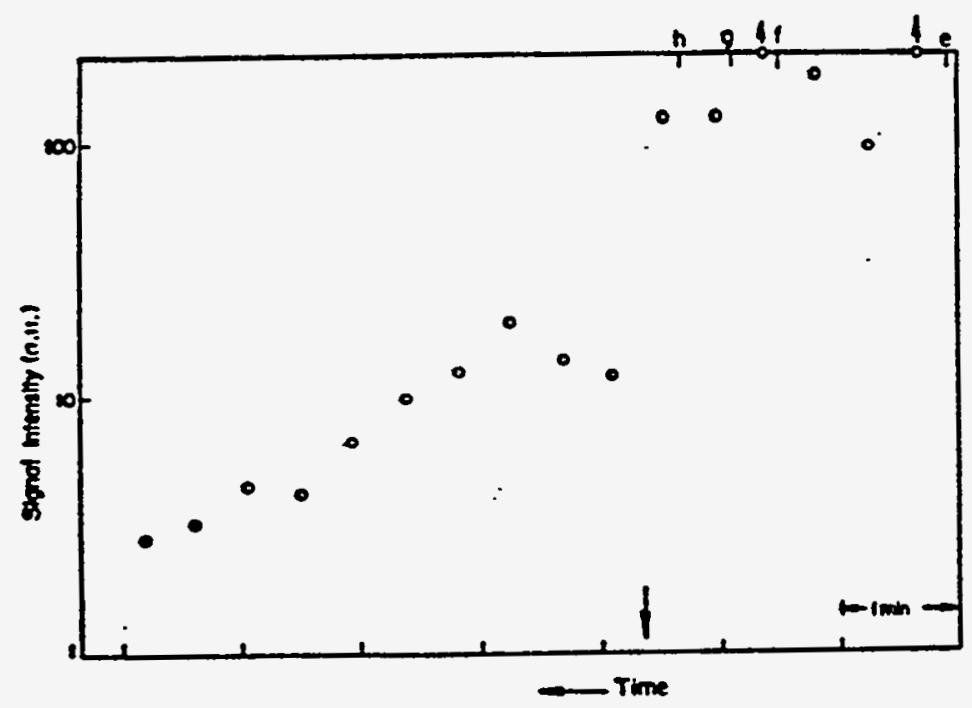

Figure 8. Intensity variation of $\mathrm{K}^{+}$signals during the field desorption process shown in Figure 7. The letters e-h denote the times of the corresponding images of Figure 7. Figure from Reference 7, reproduced with permission.

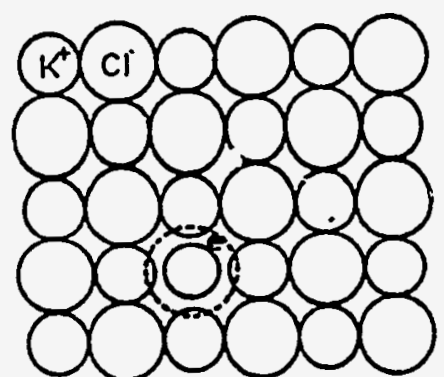

(a)

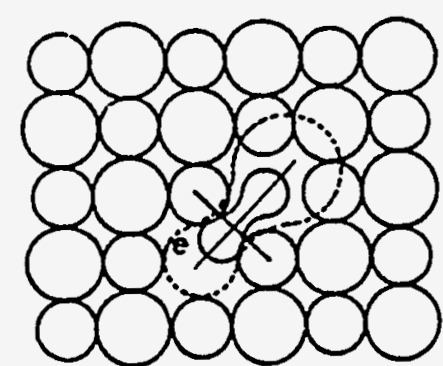

(c)

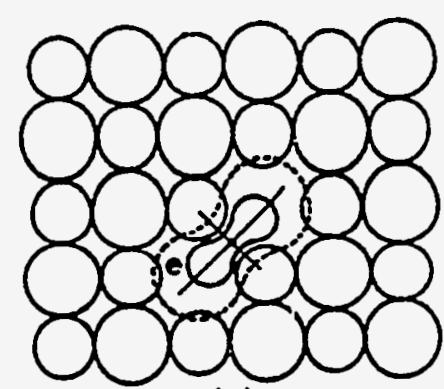

(b)

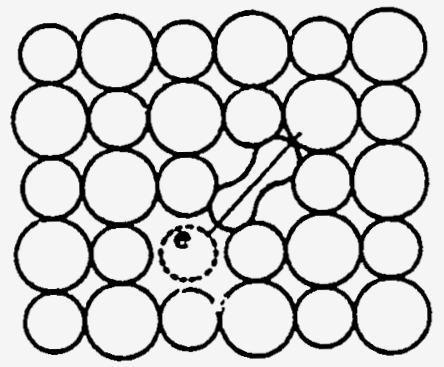

(d)

Figure 9. Excitonic defects in an alkali-halide lattice: a) unrelaxed exciton (schematically), b) self-trapped exciton, i.e. an elecron trapped by a $\mathrm{V}_{\mathrm{k}}$ center or (halogen) ${ }_{2}^{-}$ molecular ion, c) and intermediate configuration, and d) a Frenkel pair at nearest neighbor sites. Figure from Reference 10, reproduced with permission.

work function for field desorption of the ions. They note: "The typical bursts of emission observed during the FD [field desorption] of salts at low temperatures can be attributed to space charge effects i.e. to the desorption of a part of a surface layer on exceeding a critical charge density, since it is probable that the thermal activation of cations is not sufficient to overcome the work function under these conditions." [9]. 
There are four aspects of the above mechanism which are significant to our understanding of the ion formation mechanism in PBFA II: i) the emission occurs from cubic crystallites, ii) fields of the order of a few MV/cm are sufficient for field desorption. iii) the removal of the $\mathrm{Li}^{+}$ions is compensated by ionic conduction, and iv) positive charging of the emitting region is considered to lower the work function for electric field desorption.

\section{Theory of bombardment-induced electronic conductivity in LiF thin-film coatings on metal anodes}

\subsection{Introduction}

It is well known that although the diodes used in the PI-110A accelerator and PBFA II are magnetically insulated, some $\mathrm{MeV}$ electrons escape the confining field and strike the $\mathrm{LiF}$ thin film at near grazing incidence. In this section it is shown that these electrons have sufficient current densities to render the crystallites of the thin film electronically conducting on nanosecond time scales. For simplicity, the crystalline structure of the film is ignored at first and the diode is treated in a planar approximation. The analysis is described in three parts. In Section 4.2 electron transport calculations are presented which determine the amount of energy deposited in a $5000 \AA \mathrm{LiF}$ thin film on a 6-mm stainless steel substrate as a function of electron energy and incidence angle. In Section 4.3 the results of three-dimensional particle-in-cell diode simulations using the QUICKSILVER code are used to determine appropriate electron energies, intensities, and incidence angles for PBFA II. From these it is shown in Section 4.4 how to use the energy deposited per unit volume per unit time to estimate the bombardment-induced electronic conductivity and the distribution of electrons and holes in the LiF thin film. Section 4.5 is devoted to the argument that, in the PBFA II and PI-11OA diode electric fields, an electric field desorption mechanism analogous to that observed by Rollgen will occur in the electronically conducting crystallites of the LiF columnar growth. Section 4.6 takes up the generation of neutral fluorine during diode operation because it is a potentially harmful diode contaminant and because fluorine desorption is an inevitable consequence of the electron bombardment.

Our goal here is to establish an analogy between the Rollgen mechanism and the electric field desorption of $\mathrm{Li}^{+}$from the crystalline $\mathrm{LiF}$ columns in pulsed ion sources. In the ion sources, ionic conductivity is replaced by electronic conductivity. It is therefore essential to understand what are the positive and negative carriers produced by $\mathrm{MeV}$ electron bombardment in LiF [21].

The negative carrier is the electron (instead of the cation, $\mathrm{Li}^{+}$, vacancy which is produced thermally); the positive carrier is the self-trapped hole or $V_{k}$ center (instead of the anion, $\mathrm{F}^{-}$, vacancy). By definition a hole is a neutral $\mathrm{F}$ atom which results from a normal lattice $\mathrm{F}^{-}$ion when the ion's electron is removed into the conduction band and becomes the delocalized electron of an electron-hole pair. As discussed in [21] it is assumed here that in $\mathrm{LiF}$ this neutral $\mathrm{F}$ atom associates itself immediately with one neighboring $\mathrm{F}^{-}$ion to form 
a $\mathrm{F}_{2}{ }^{-}$molecular ion, essentially the same ion which can occur in free space [21]. This $\mathrm{F}_{2}{ }^{-}$ ion is not very mobile and is known as a self-trapped hole or $V_{k}$ center [21]. The selftrapped hole attracts any conduction band electron and within a picosecond the electron and self-trapped hole can recombine to make what is known as a self-trapped exciton. ${ }^{*}$ A subsequent electronic decay process results in the production of nearest-neighbor $F$ center$\mathrm{H}$ center defect pairs. ${ }^{\dagger}$ At room temperature most of these annihilate and heat the crystal locally; about $7 \%$ of the time the $\mathrm{F}$ and $\mathrm{H}$ centers separate and survive as independent entities. The excitonic phases of these lattice defect processes are illustrated in Figure 9. In Figure 9(a) an electron (the dotted circular orbit) and a neutral halogen atom are shown bound together as a free exciton. These are not observed in $\mathrm{LiF}$ at room temperature. The $\mathrm{F}$ atom is a hole before self-trapping. Part (b) of Figure 9 shows a self-trapped exciton in its initial stage. Its core is a $V_{k}$ center, and the electron orbits about this diatomic molecule $\left(\mathrm{Cl}_{2}{ }^{-}\right.$in Figure 9). The $\mathrm{V}_{\mathrm{k}}$ center by itself is of particular importance here because, as will be seen in Section 4.4, the ion source electric field can sweep the electrons away from the self-trapped holes before recombination can occur. The $V_{k}$ center is the positive carrier in our analogy and the electron is of course the negative carrier. When recombination occurs, a sub-picosecond evolution from Figure 9(b) through (c) to (d) takes place, yielding the nearest neighbor $\mathrm{F}$ center $\mathrm{H}$ center pair. The $\mathrm{F}$ center is the electron bound in an anion vacancy; the $\mathrm{H}$ center is the same molecular ion as the $\mathrm{V}_{\mathrm{k}}$ center, but squeezed into the site occupied normally by a single negative ion. The $\mathrm{H}$ center thus incorporates an interstitial neutral halogen. The $\mathrm{H}$ center and $\mathrm{F}$ center have the proper charge for their lattice sites so they are not pulled around by external electric fields as are the electron and the $V_{k}$ center.

We can draw an analogy between steady-state, thermally-induced operation (in mass spectrometry applications) and nanosecond response (in pulsed ion sources) due to the high mobility of the electron. The arrival of the negative cation vacancies at the tungsten wire in the steady-state experiments is replaced by the barrierless passage of the electrons into the anode metal conduction band. The surface positive charging which arises out of bombardment-induced conductivity will be seen to occur as the result of electron-hole pair formation in the presence of the diode electric field. This produces a region of $\mathrm{V}_{\mathrm{k}}$ centers within about $5 \mathrm{~nm}$ of the emitting surface, as will be shown in Section 4.4

\subsection{Electron and photon transport in the LiF-coated PBFA II anode}

In this section, the energy deposited in the $\mathrm{LiF}$ as the result of $\mathrm{MeV}$ electron bombardment is determined. Discrete ordinate adjoint transport calculations were carried out for $500 \mathrm{~nm}$ of LiF on $6 \mathrm{~mm}$ of stainless steel (SS) [22]. These are planar-slab calculations which allow the incident monoenergetic electron to enter at select angles $\theta$ with respect to

\footnotetext{
${ }^{*}$ A newly formed hole can propagate as a valence band particle for some distance before self-trapping takes place. This propagation takes place on picosecond time scales. The mean free path for self-trapping in LiF is not known at present but could be some tens of Angstroms. In our discussion, self-trapping will be assumed to occur immediately.

'The term "F center" denotes an F (Farben) center, and is not to be confused with the chemical symbol $\mathrm{F}$ for fluorine. Fluorine will be referred to as " $\mathrm{F}$ atom" or " $\mathrm{F}$ - ion".
} 


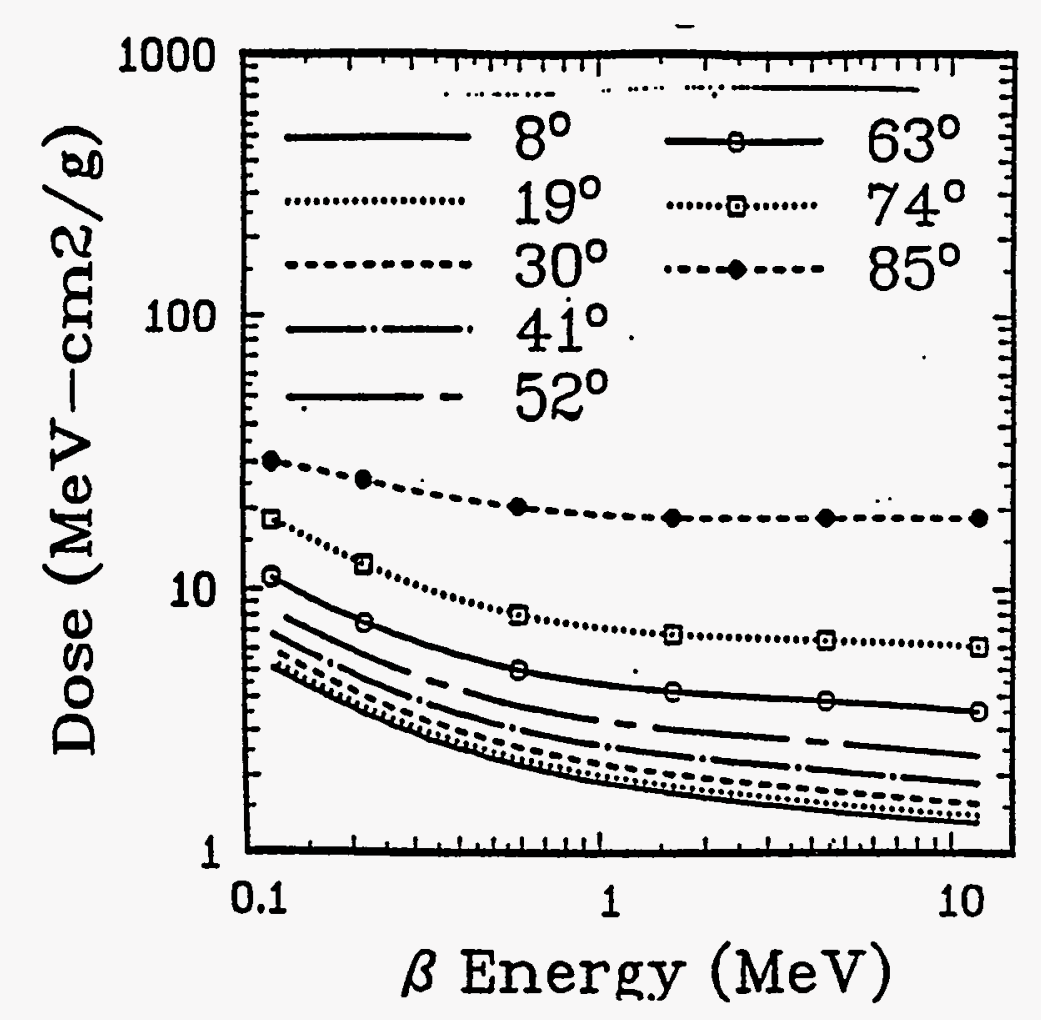

Figure 10. Mono-energetic $\beta$ incident on LiF-coated stainless steel, showing Dose delivered into $5000 \mathrm{~A}$ of LiF.

the normal of the anode plane. Figure 10 summarizes the main results for incident energies between $0.12 \mathrm{MeV}$ and $12 \mathrm{MeV}$ and for a selection of angles of incidence. The ordinate, Dose, is equal to the average energy deposited per unit length in the $\mathrm{LiF}$ thin film divided by the density, $\rho$, of $\operatorname{LiF}\left(p=2.635 \mathrm{~g} / \mathrm{cm}^{3}\right)$. The deposited energy in the film, whose thickness $d=5 \times 10^{-5} \mathrm{~cm}$, is obtained by multiplying Dose by $\rho d$. We will use the results for $\theta=85$ because this value is consistent with QUICKSILVER simulations reported in Section 4.3. As would be expected, the larger the value of $\theta$ the longer the path of the incident electron in the LiF thin film and the larger the deposited energy. Note that the deposited energy is not very dependent on the incident electron $(\beta)$ energy, even down to the energies characteristic of the PI-110A accelerator. From Figure 10 we will adopt a Dose value of $19 \mathrm{MeV}$ $\mathrm{cm}^{2} / \mathrm{gm}$. To about 1 part in 1000 this is equivalent to taking $2500 \mathrm{eV}$ as the amount of energy deposited in the LiF thin film per incident $\beta$.

The quantity Dose relates to energy loss experienced in the $\mathrm{LiF}$ medium by a beam of fast electrons, taking into account the effect of the stainless steel substrate as well as electron loss. It is a local variable in terms of which the rate of energy deposition per unit volume, $R_{V}$, is given by

$$
R_{V}\left[\frac{\mathrm{MeV}}{\mathrm{cm}^{3} \mathrm{sec}}\right]=\rho \cdot \Phi_{e} \cdot \text { Dose }
$$


Here $\Phi_{\mathrm{e}}$ is the particle flux (i.e. the number of particles crossing a unit area per sec). It can be expressed as the product of the beam particle density $n_{e}$, and the particle velocity $v_{e}$.

Additional calculations of backscattered electron angle and energy distributions (not reported here) uncovered an important aspect of the backscattered electron distribution for $12 \mathrm{MeV} \beta$ particles incident at $\theta=85^{\circ}$ on the $\mathrm{LiF} / \mathrm{SS}$ anode. It was found that with a probability of about 0.50 electrons are quasielastically scattered back out of the stainless steel through the $\mathrm{LiF}$ film into the anode-cathode (AK) gap at about the same $85^{\circ}$ angle of the incident electron. Only 5.3 MeV of the initial $12 \mathrm{MeV}$ is deposited in the anode and $5.9 \mathrm{MeV}$ is carried away by the reflected electrons. This is due to Coulomb scattering by the iron and other heavy elements in the stainless steel. The calculations also show that almost $1 \mathrm{MeV}$ has been converted into photons. In PBFA II these backscattered electrons are returned to the anode by the large diode electric field for a second, third, etc. passage into the stainless steel. In this way the energy deposited in the LiF thin film per incident $\beta$ (at $\theta=85^{\circ}$ ) should be approximately doubled. For this reason the energy deposition values given by Figure 10 are doubled in our application.

\subsection{QUICKSILVER particle in cell simulations of a PBFA II shot}

It is necessary to know what electron energy and angle $\theta$ to use with the results from Section 4.2. Although ion diode simulations with the QUICKSILVER code are used primarily for other purposes [16][17], the QUICKSILVER code [15] provides our best theoretical tool for getting information about the electron currents onto and near the anode. Figure 11(a) shows the energy and angle distribution of electrons striking the anode as an intensity contour plot for an electron-only simulation. In this simulation, no $\mathrm{Li}^{+}$ions were emitted from the anode. The distribution corresponds to a time $15 \mathrm{~ns}$ after the arrival of the voltage pulse at the diode. By this time the diode is "saturated". This means that the cloud of electrons emitted from the cathode have filled the diode gap. Figure 11(b) shows a qualitatively similar distribution for a simulation which includes the emission of $\mathrm{Li}^{+}$ions. The figure shows that the leakage electrons which strike the anode are primarily multi- $\mathrm{MeV}$ electrons and that they have a typical incidence angle between $80^{\circ}$ and $85^{\circ}$ from the surface normal. The calculations also provide values for the leakage electron current density as a function of time. It increases substantially when the diode saturates and is still larger when the $\mathrm{Li}^{+}$ions are included in the simulation. A value of about $150 \mathrm{~A} / \mathrm{cm}^{2}$ would be appropriate for the simulation which includes the $\mathrm{Li}^{+}$ions. The results from the $\mathrm{Li}$ simulations will provide guidance for numerical estimates in the following sections.

It should be pointed out that the simulation of $\mathrm{Li}^{+}$ion emission shown in Figure 11(b) is based on a space-charge-limited anode boundary condition which maintains the normal component of the electric field equal to zero at the anode. The anode electric field then rises rapidly to high values very close to the anode. The electron-only simulation does not include this additional boundary condition. For field desorption, the field at the anode surface must be non-zero. On the basis of the similarity of parts (a) and (b) In Figure 11, we do not believe that the introduction of an appropriate boundary condition for a field desorption source would change the leakage electron energy and angular distributions signifi- 
a)

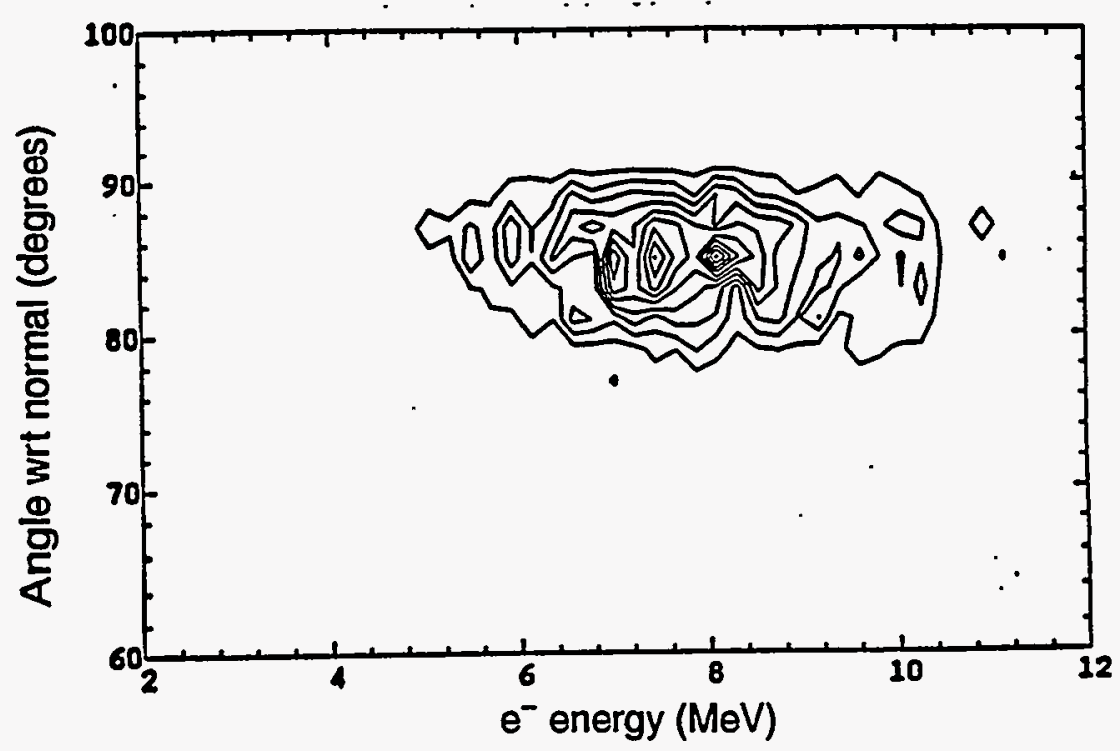

b)

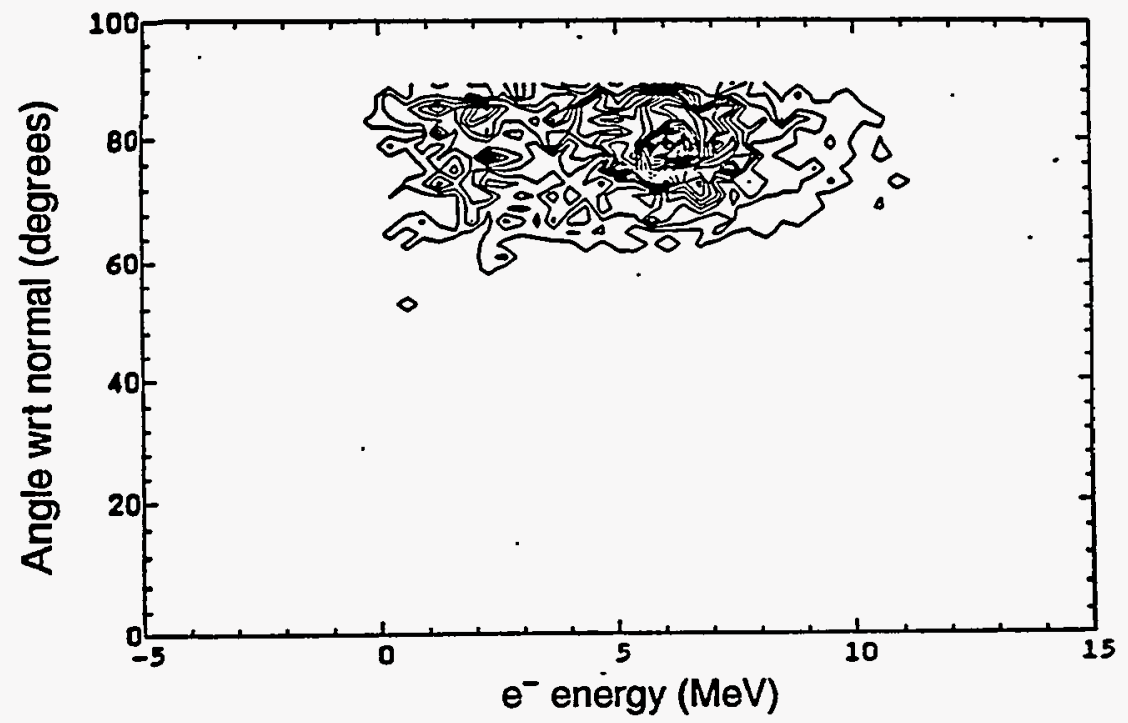

Figure 11. Energy-angle distributions of electrons striking the anode from QUICKSILVER simulations including a) only electrons and b) both electrons and $\mathrm{Li}^{+}$ions. Electron sheath is saturated in the simulations at times of these distributions.

cantly. In addition, as shown in Figure 10, the electron deposition for $85^{\circ}$ is insensitive to the electron energy.

\subsection{Bombardment-induced heating, electronic conductivity, and surface charging in the presence of an external electric field}

We wish to show that the $\mathrm{LiF}$ anode thin films are electronically conducting and that their field-emitting surfaces charge up positively in the PI-110A and PBFA II accelerators. 
Let the leakage current (in $\mathrm{A} / \mathrm{cm}^{2}$ ) be designated as $J_{x}$, and let the amounts of deposited energy (in $\mathrm{eV}$ ) necessary to make an electron-hole (e-h) pair and a separated $\mathrm{F}$ center $\mathrm{H}$ center $(\mathrm{F}-\mathrm{H})$ pair be designated as $W_{e h}$ and $W_{F H}$ respectively. Let the density of $\mathrm{LiF}$ (in $\mathrm{gm} / \mathrm{cm}^{3}$ ) be $\rho$, and let the thickness of the LiF thin film (in $\mathrm{cm}$ ) be $d$. Let the specific heat of LiF (in J/gm-deg) be designated as $C_{p}$ and let the time during which the anode is irradiated by the leakage electrons be $\tau$ sec. For these estimates, the values discussed in Sections 4.2 and 4.3 will be used. For LiF, $p=2.635 \mathrm{gm} / \mathrm{cm}^{3}, C_{p}=1.561 \mathrm{~J} / \mathrm{gm}-\mathrm{deg}, W_{e h}=35 \mathrm{eV}$, and $W_{F H}=500 \mathrm{eV}$. For our estimates we will use $J_{\chi}=1000 \mathrm{~A} / \mathrm{cm}^{2}$, a value suggested by subtracting the diode ion current from the total diode current in typical PBFA II shots. This is probably an upper bound and it is quite a bit larger than the theoretical value reported in

Section 4.3. Our estimates will be reported in terms of $J_{x} / 1000$ so they can be easily scaled to other values. Let the rate of energy deposition per unit volume (in $\mathrm{J} / \mathrm{cm}^{3}-\mathrm{sec}$ ) be designated as $R_{V}$. Then, from (1) with Dose $=2 \times 19 \times 10^{6} \mathrm{eV}-\mathrm{cm}^{2} / \mathrm{gm}$, the rate of temperature rise $d T / d t$ is given by

$$
\frac{d T}{d t}\left[{ }^{\circ} \mathrm{C} / \mathrm{sec}\right]=2.43 \times 10^{10}\left(\frac{J_{x}}{1000}\right)
$$

The temperature rise in $15 \mathrm{~ns}, \Delta T(15 \mathrm{~ns})$ is then

$$
\Delta T(15 n s)\left[{ }^{\circ} \mathrm{C}\right]=1.5 \times 10^{-8} \frac{d T}{d t} \approx 365\left(\frac{J_{x}}{1000}\right)
$$

Since the melting temperature of $\mathrm{LiF}$ is $870^{\circ} \mathrm{C}$, there is no reason to expect that leakage electron bombardment in PBFA II would melt the LiF coating.

An analogous approximate calculation can be carried out for the stainless steel. The result is $\Delta T(15 \mathrm{~ns} / \mathrm{SS}) \approx 576\left(J_{x} / 1000\right)^{\circ} \mathrm{C}$. This estimate does not allow for the cooling effect of heat conduction. Stainless steel melts at $1427^{\circ} \mathrm{C}$.

We next consider the production of e-h pairs and separated F-H pairs in the thin film. Using a deposited energy of $5000 \mathrm{eV}$ per incident-electron from Section 4.2, the efficiencies are simply $5000 / 35=143 \mathrm{e}-\mathrm{h} / \mathrm{\beta}$ and $5000 / 500=10 \mathrm{~F}-\mathrm{H} / \beta$ respectively. Now $1000 \mathrm{~A} /$ $\mathrm{cm}^{2}$ of electrons corresponds to $6.24 \times 10^{21} \beta / \mathrm{cm}^{2} / \mathrm{sec}$. Dividing by $\mathrm{d}=5 \times 10^{-5} \mathrm{~cm}$, the volume rates of production of e-h and separated F-H pairs are

$$
\begin{aligned}
& R_{e h}=1.78 \times 10^{28}\left(\frac{J_{x}}{1000}\right) \text { e-h pairs } / \mathrm{cm}^{3} / \mathrm{sec} \\
& R_{F H}=\frac{35}{500} R_{e h} \text { separated F-H pairs } / \mathrm{cm}^{3} / \mathrm{sec} .
\end{aligned}
$$

$R_{e h}$ can equally well be calculated as $R_{V} / W_{e h}$ using (1). This results in $R_{e h}=1.79 \times 10^{28}\left(J_{x} J\right.$ 1000) e-h pairs $/ \mathrm{cm}^{3} / \mathrm{sec}$. 
These quantities are the source terms in the kinetic equations for the production and loss of these defects [23]. At e-h pair densities above $10^{16} \mathrm{~cm}^{-3}$, binary recombination of electrons and $V_{k}$ centers to form self-trapped excitons is the dominant electron and hole loss mechanism [23]. If $n_{e}$ stands for the density of electrons in the conduction band (it is equal to the number of $V_{k}$ centers here), and $\alpha$ is the recombination coefficient,

$$
\frac{d n_{e}}{d t}=R_{e h}-\alpha n_{e}^{2}
$$

From this the equilibrium density $\dot{n}_{\text {inf }}$ of electrons is given by

$$
n_{\text {inf }}=\sqrt{\frac{R_{e h}}{\alpha}} .
$$

By scaling (6) with $n_{\text {inf }}$ we can write

$$
\frac{d u}{d s}=X-u^{2}
$$

where $u=n_{e} / n_{\text {inf }}, X=R_{e h} /\left(\alpha n_{\text {inf }}^{2}\right)$, and $s=t \sqrt{\alpha R_{e h}}$. Thus the equilibrium electron (and $\mathrm{V}_{\mathrm{k}}$ center) concentration is reached at the rate $r_{b}=\sqrt{\alpha R_{e h}}$. The binary rate constant $\alpha$ has not been measured for LiF. We shall use the value $10^{-14} \mathrm{~cm}^{2}$ for the electron-hole recombination cross-section because it is of the same order of magnitude as the experimental value for $\mathrm{KCl}$ [23]. We shall also estimate the electron speed, $v_{e}$, at the bottom of the conduction band to be $10^{7} \mathrm{~cm} / \mathrm{sec}$ [23]. The rate coefficient, $\alpha$, is equal to $v_{e}$ times the cross section and therefore has the estimated value $10^{-7} \mathrm{~cm}^{3} / \mathrm{sec}$. Then

$$
\begin{aligned}
& n_{\text {inf }}=4 \times 10^{17} \sqrt{\frac{J_{x}}{1000}} \mathrm{e} / \mathrm{cm}^{3}, \\
& r_{b}=4 \times 10^{10} \sqrt{\frac{J_{x}}{1000}} \sec ^{-1} .
\end{aligned}
$$

It is important in connection with the PI-110A machine to note that equilibrium is reached on a nanosecond time scale even if $J_{x}$ is only $10 \mathrm{~A} / \mathrm{cm}^{2}$.

The electronic conductivity under steady state leakage electron current bombardment is given by $\sigma_{e}=e n_{\text {inf }} \mu_{e}$, where $\mu_{e}$ is the electron mobility and $e$ is the electron charge. The mobility has not been measured for LiF. Values from the alkali-halide literature [24] suggest that a value near $10 \mathrm{~cm}^{2} /$ volt-sec be used. With $\mu_{e}=10^{-3} \mathrm{~m}^{2} /$ volt-sec,

$$
\sigma_{e}=70 \sqrt{\frac{J_{x}}{1000}} \mathrm{ohm}^{-1} \mathrm{~m}^{-1}
$$


which is more than a million times the ionic conductivity near $375^{\circ} \mathrm{C}$. It should be kept in mind, however, that the conductivity exhibited in (11) is still less that $10^{-4}$ of that possessed by the underlying stainless steel. The dielectric relaxation rate (using $\kappa_{e}=9.27$ ) is

$$
\frac{\sigma_{e}}{\kappa_{e} \varepsilon_{o}}=8 \times 10^{11} \sqrt{\frac{J_{x}}{1000}} \sec ^{-1}
$$

The foregoing discussion does not take into account the fact that the generation of electron-hole (e-h) pairs takes place in an electric field. For a $10 \mathrm{MV} / \mathrm{cm}$ field in the AK gap near the anode, the dielectric polarization in a planar geometry reduces the field by a factor 9.27 in the interior of the LiF thin film. This internal field is still very large however and can influence the recombination of e-h pairs by rapidly pulling electrons toward the metallic anode which they can enter without any barrier. There should be no barrier because the affinity of the LiF is very likely to be less than $1 \mathrm{eV}$ and therefore less than the work function of the stainless steel substrate. Thus, as a result of the internal electric field, an excess of $V_{k}$ centers and a deficit of electrons will be produced near the vacuum interface of the $\mathrm{LiF}$ thin film. As a result, the film develops a positive charge on the vacuum interface. This is important because, in the mass spectrometry experiments described in Section 3, surface charging lowered the work function for field desorption from the ionically conducting crystals. It will now be shown that the positive $V_{k}$ center layer at the surface - the layer which reduces the electric field to zero in the rest of the $\mathrm{LiF}$ - is only about $5 \mathrm{~nm}$ thick.

The one-dimensional treatment which will be used can only apply in an approximate sense to the crystallites which comprise the actual anode thin film. However, a one-dimensional treatment with an electric field normal to the thin film illustrates the essential physics. Let us reconsider the electron and hole kinetics in an electric field in one dimension. Using the same notation as in (6), letting $\phi$ stand for the electric potential, and letting distance $x$ increase from zero as one moves from the vacuum interface into the LiF thin film, we have

$$
\begin{gathered}
\frac{d n_{e}}{d t}=R_{e h}-\alpha n_{e} n_{h}-\frac{d}{d x}\left[-D_{e} \frac{d n_{e}}{d x}+n_{e} \mu_{e} \frac{d \phi}{d x}\right] \\
\frac{d n_{h}}{d t}=R_{e h}-\alpha n_{e} n_{h} \\
-\frac{d^{2} \phi}{d x^{2}}=\frac{e\left(n_{h}-n_{e}\right)}{\varepsilon}
\end{gathered}
$$

In the kinetic equations (13) and (14), the diffusive and electric-field-induced contributions to the electron current per unit charge have been introduced. The self-trapped holes are taken to be immobile on PBFA II time scales in comparison with the electrons. The potential is related to the charge density by Poisson's equation (15), where $\varepsilon$ is the LiF permittivity. As with (6) we look for a steady-state solution with a charge density 
approaching zero in the interior of the LiF. In this limit $n_{e}=n_{h}=\sqrt{R_{e h} / \alpha}$ as in (7). From (14), in the steady state for all $x, R_{e h}=\alpha n_{e} n_{h}$. Consequently, from (13) the term (dl $d x)[. . .$.$] must vanish. This term represents the divergence of the electron current density per$ unit charge. Consider the case where the $\mathrm{LiF}$ film has acquired a charge, no $\mathrm{Li}^{+}$ions are being emitted, and the flow of electron current has ceased so that the term [...] in (13) is zero. This means that

$$
\frac{d}{d x} \ln n_{e}=\frac{\mu_{e}}{D_{e}} \frac{d \phi}{d x}
$$

and

$$
n_{e}=n_{o} \exp \left(\frac{\mu_{e} \phi}{D_{e}}\right)
$$

where $\mu_{e}$ is the electron mobility, $D_{e}$ is the electron diffusion constant, and $n_{o}$ is a constant of integration. We want to choose $n_{o}$ so that deep in the LiF film $n_{e}$ and $n_{h}$ approach $n_{i n f}$ of (7). This is accomplished by writing, following Kliewer [25]

$$
\begin{gathered}
n_{e}=n_{i n f} \exp Z \\
n_{h}=n_{i n f} \exp (-Z)
\end{gathered}
$$

where the potential is expressed in terms of the variable $Z$ through

$$
Z=\frac{e}{k T}\left(\phi-\phi_{i n f}\right)
$$

Here, $n_{\text {inf }}$ is given by (7) and $\phi_{\text {inf }}$ comes from the constant of integration in (15). It has been chosen to correspond to the potential deep in the LiF layer when the potential is zero at the $\mathrm{LiF}$-vacuum interface. Thus as $Z$ goes to zero, $\phi$ goes to $\phi_{i n f}, n_{e}$ and $n_{h}$ go to $n_{\text {inf }}$, and the charge density goes to zero in (15). The factor $e / k T$, where $k$ is the Boltzmann constant and $T$ is the absolute temperature, comes from the Einstein relation $\mu_{e} / D_{e}=e / k T$. Poisson's equation (15) can now be written in the form

$$
\frac{d^{2} Z}{d s^{2}}=\sinh Z
$$

where $s=\kappa x$ and

$$
\kappa^{2}=\frac{2 e^{2} n_{i n f}}{\varepsilon k T} .
$$


The inverse of $\kappa$ determines the size of the region adjacent to the vacuum where $n_{h}$ exceeds $n_{e}$ and the internal electric field is non vanishing. Beyond $x=1 / \mathrm{k}$ the electric field goes rapidly to zero. It is the value of $\kappa$ which we seek. Again following Kliewer, when the film thickness $d$ is large compared to $1 / \kappa$, the solution of (21) can be written as

$$
Z=4 \operatorname{atanh}\left(\exp (-s) \tanh \left(\frac{Z_{0}}{4}\right)\right)
$$

where $Z_{o}$ is the value of $Z$ when $x$ (and $s$ ) are zero at the vacuum interface. Since we have chosen the potential $\phi$ to be zero at the vacuum interface where $x$ and $s$ are zero, it follows from (23) and (20) that $Z_{o}=-\left(e \phi_{\text {inf }}\right) /(k T)$. The $x$-component of the electric field, $E_{x}$, is equal to $-d \phi / d x$. Since $\phi(x)=(k T / e) Z(x)+\phi_{\text {inf }}$, the electric field can be seen to vanish exponentially with increasing $s$ and $x$ as one moves into the $\mathrm{LiF}$ film. The value of the interior field at the vacuum interface is found by differentiating (23), and is given by

$$
E_{x}(x=0)=\frac{2 \kappa k T}{e} \sinh \left(\frac{Z_{0}}{2}\right)
$$

The interior value of the electric displacement $D_{x}$ is given by $\varepsilon E_{x}$. The displacement is continuous through the interface with the vacuum and so the external electric field of magnitude $E_{o}$ in the vacuum, just outside the $\mathrm{LiF}$ thin film, is given by

$$
E_{o}=-\left(\frac{\varepsilon}{\varepsilon_{0}}\right) E_{x}(x=0)
$$

From (25), given the external field $E_{o}$, one can find the values for $E_{x}(x=0)$ in the interior and $Z_{o}$ from the last two equations. Everything else is then determined. The total surface charge per unit area, $Q_{A}$, from the flow of electrons into the stainless steel substrate during the charging process is given by Gauss' law and is equal to $-\varepsilon E_{x}(x=0)$.

It is now possible to draw some interesting conclusions about the bombarded LiF layer. Consider an external vacuum electric field of $10^{7} \mathrm{~V} / \mathrm{cm}$, and let $n_{\text {inf }}=4 \times 10^{17} \mathrm{~cm}^{-3}$ as suggested by (9). Then $E_{x}(x=0)=-1.1 \times 10^{6} \mathrm{~V} / \mathrm{cm}$ and $\kappa=1.9 \times 10^{6} \mathrm{~cm}^{-1}$. The inverse of $\kappa$ is equal to $5.2 \mathrm{~nm}$, showing that the positively charged region is thin compared to the film thickness we have assumed. We also find $Z_{o}=-5.2, \phi_{i n f}=0.22 \mathrm{~V}$, and $Q_{A}=8.85 \times 10^{-3} \mathrm{C} /$ $\mathrm{m}^{2}=5.53 \times 10^{12} \mathrm{e} / \mathrm{cm}^{2}$. This is of course the charge required to screen out $E_{o}$ deep in the interior (several times $\kappa$ ) of the conducting LiF thin film. The density, $n_{h}$, of $V_{k}$ centers at the vacuum interface is given by (19). Since $\exp \left(-Z_{o}\right)=175$ the concentration of $V_{k}$ centers near the surface is high compared to that in the neutral region of the LiF thin film. The electric field acting on the LiF thin film has swept electrons away from the vacuum interface and produced a preponderance of positive $V_{k}$ centers within about $5 \mathrm{~nm}$ of the surface. Using the value $R_{e h}=1.8 \times 10^{19} \mathrm{e}-\mathrm{h}$ pairs $/ \mathrm{cm}^{3} \mathrm{~ns}$ from (4) and $\kappa^{-1}=5.3 \times 10^{-7} \mathrm{~cm}$, it takes approximately $Q_{A} /\left(R_{e h} \kappa^{-1}\right)=0.6 \mathrm{~ns}$ for the film to acquire a net charge.

When $\mathrm{Li}^{+}$ions are being field desorbed, the situation is more complex. First, we expect that field desorption takes place from the crystallites of the columnar growth, for which a 
one-dimensional slab approach is not realistic (even though the expected thickness of the $\mathrm{V}_{\mathrm{k}}$ center layer of positive charge is small compared to the dimensions of the crystallites). The conducting cubic crystallites will concentrate the average anode electric field, and ions will desorb from their regions of highest curvature. A solution of this more complex problem has not been attempted. However the following evolution seems plausible. The loss of $\mathrm{Li}^{+}$ions will produce a population of negative cation vacancies at the vacuum interface simultaneous with the bombardment-induced electron-hole pair generation. In the anode electric field, electrons will leave the $\mathrm{LiF}$ and enter the stainless steel conduction band and the population of positive $V_{k}$ centers produced with these electrons will increase to compensate the growth of the negative cation vacancy population. If the net positive charge at the surface is decreased too much by $\mathrm{Li}^{+}$field desorption, the $\mathrm{Li}^{+}$field desorption will decrease until sufficient bombardment-induced positive charge is generated and has lowered the work function for $\mathrm{Li}^{+}$emission. Nanosecond ion emission oscillations may perhaps occur as has been observed on much slower time scales in connection with Rollgen's experiments (see Section 3 ). The $V_{k}$ center - cation vacancy double layer near the vacuum interface has an internal electric field which favors sweeping electrons out of this region and sweeping the holes to the surface. An electron current flows through the LiF to compensate the $\mathrm{Li}^{+}$current. The bombardment-induced electronic conductivity allows this electron flow to occur without a significant voltage drop. Experimental studies of this complex process are clearly needed.

\subsection{Recapitulation of the analogy between the electric field desorption in the LiF ion source and in the Rollgen experiments}

In Sections 4.2-4.4 a quantitative argument is given which demonstrates that in the PI$110 \mathrm{~A}$ accelerator and in PBFA II there is sufficient bombardment-induced conductivity to allow electron current flow through the LiF thin film into the metal anode substrate and to create a positive $5 \mathrm{~nm}$ thick region of $\mathrm{V}_{\mathrm{k}}$ centers at the emitting surfaces of the crystallites of the LiF columnar thin film. The response of the electron-hole pair production mechanism was shown to have a subnanosecond time constant and the dielectric relaxation time was correspondingly short. We now argue on this basis that the field desorption mechanism observed for ionically conducting crystallites in $\mathrm{MV} / \mathrm{cm}$ fields will also occur in the electronically conducting crystallites of the columnar growth which constitutes the $\mathrm{LiF}$ anode thin films in PBFA II and the PI-110A accelerator.

Returning to the four aspects of the ionic conductivity mechanism in Section 3, we note the following analogies between the ionic and electronic mechanisms. First, since the emission is from cubic crystallites in both cases, the field enhancement properties of the emitters should be similar in both cases. This allows the field emission process to operate with similar external electric fields in the two cases. Second, the measured electric field in PBFA II and the structurally-enhanced electric field in the PI-110A machine were of the order of magnitude of $5 \mathrm{MV} / \mathrm{cm}$. These are fields of the same magnitude as the electric fields used in the Rollgen experiments. Third, the bombardment-induced conductivity is sufficient to generate electronic conductivity on the nanosecond time scales required for the accelerator 
ion sources. Fourth, it has been shown that the positive layer of $V_{k}$ center charge is concentrated within $5 \mathrm{~nm}$ of the ion emitting regions. This should allow field desorption of $\mathrm{Li}^{+}$ ions to be aided - through a lowering of the work function for field desorption - just as in the case of charging via ionic conduction.

This concludes our argument for an analogy between the two mechanisms. The large current densities measured in the accelerators are the result of the high density of crystallites per square $\mathrm{cm}$ in the anode columnar thin films, as discussed in Section 2.3.

We conclude this section with a comment about fluorine atom production. In the case of ionic conduction, the positive charges are anion vacancies and the negative charges are cation vacancies. When a $\mathrm{Li}^{+}$ion leaves into the vacuum, the cation vacancy it leaves behind must migrate toward the tungsten wire, providing an ionic current flow as in electrolysis. At the tungsten wire an F ion is neutralized. The net result is the loss of one LiF molecule from the crystallite. In PBFA II, an electron goes to the metal anode when a $\mathrm{Li}^{+}$ ion is field-desorbed into the vacuum. According to the picture outlined above, a thin double layer of $\mathrm{V}_{\mathrm{k}}$ centers and cation vacancies is formed. From the macroscopic point of view, the positive charge represented by the self- trapped hole is little different from that of a missing halogen ion or anion vacancy. In each case one negative charge is missing from the normal lattice. However the $V_{k}$ center includes a neutral $F$ atom. Diffusion of the $V_{k}$ centers toward the vacuum interface can produce surface $V_{k}$ centers which macroscopically neutralize the negative charge of the cation vacancies on the surface, reducing the double layer and its attendant electric field. It is important to know if aggregates of $V_{k}$ centers and cation vacancies can interact to release fluorine atoms at the surface. In this quite-possible event the emission of a monolayer of $\mathrm{Li}^{+}$ions could be accompanied by up to a monolayer of fluorine atoms. This would be highly undesirable in PBFA II. The loss of fluorine, then, is quite different in the two mechanisms. In the case of ionic conductivity, fluorine loss occurs at the metal substrate; in the case of electronic conductivity the fluorine loss would occur at the vacuum interface. This distinction merits further experimental study.

\subsection{Electron-bombardment-induced neutral ground-state fluorine atom production in PBFA II}

In the previous section the possibility has been raised that fluorine atoms desorb from surface $V_{k}$ centers in the midst of cation vacancies. There is yet another (well established) source of fluorine atoms from the electrically neutral regions of a bombarded crystallite. In these regions electron-hole pair recombination balances production according to (6). At room temperature and above, a fraction of the recombining electron-hole pairs leads to the generation of $\mathrm{F}$ center - $\mathrm{H}$ center pairs, as described by (5). An $\mathrm{H}$ center incorporates an interstitial $\mathrm{F}$ atom while an $\mathrm{F}$ center is an electron in the halogen site left by the interstitial $F$ atom, as discussed in Section 3 . The $F$ centers are immobile on PBFA II time scales. It is known from experiment that when an $\mathrm{H}$ center arrives at a crystal surface it releases its interstitial $\mathrm{F}$ atom which then desorbs [21]. The amount of desorbed fluorine from this mechanism is estimated below. The effect of the electric field in producing a thin layer of $\mathrm{V}_{\mathrm{k}}$ centers near the vacuum interface will be ignored and a uniform production of separated 
$\mathrm{F}$ centers and $\mathrm{H}$ centers will be assumed. In Section 4.4 it was shown that each incident $\mathrm{MeV} \beta$ particle could be expected to produce 10 separated $\mathrm{F}$ center $-\mathrm{H}$ center pairs in a $5000 \AA$ film on stainless steel. That is, if all the $F$ atoms produced by a $1000 \mathrm{~A} / \mathrm{cm}^{2}$ leakage current density escaped into the AK gap, the particle flux of fluorine atoms would be the equivalent of $10 \mathrm{kA} / \mathrm{cm}^{2}$. However, the diffusion coefficient of the $\mathrm{H}$ center is not likely to be large enough to allow the majority of the $\mathrm{H}$ centers to reach the thin film surface on time scales of $15 \mathrm{~ns}$. The H-center diffusion coefficient hasn't been measured for LiF and so our estimate will be based on a value for $\mathrm{NaCl}$ derived from colloidal metal generation by Hughes and Lidiard [26]. Using this diffusion coefficient we find that the distance traveled in a one-dimensional diffusion process by an $\mathrm{H}$ center in $15 \mathrm{~ns}$ is $50 \mathrm{~nm}$. Thus for a $500 \mathrm{~nm}$ film one can estimate that about $10 \%$ of the $10 \mathrm{H}$ centers, or one $\mathrm{H}$ center per incident electron, can release a fluorine atom into the AK gap.

The generation of neutral fluorine during $\mathrm{Li}^{+}$ion generation is important because it can contribute to the contamination of the ion source in the same way as the desorption of adsorbed foreign species.

\section{Flashover and dielectric breakdown on a LiF- coated anode in PBFA II}

\subsection{Introduction}

The most widely used and most popular ion source for magnetically insulated diodes is the flashover source. A review of these and other sources which have been studied at Sandia has been given by R. A. Gerber [1]. Flashover sources are also discussed in Ref. 27. The essential feature of a flashover source is the rapid formation of a plasma on the anode surface. The anode material is incorporated into the plasma from which the ions are drawn. Such a plasma screens the anode surface from the diode electric field and would prevent field emission processes from occurring. Under these circumstances it is essential to devote some attention to the question of whether early on in the diode power pulse flashover and/ or dielectric breakdown in the $\mathrm{LiF}$ thin film can occur. If a plasma can be shown to form early in the power pulse which will reduce the electric field on the $\mathrm{LiF}$ to very low values, then the whole field desorption picture developed here becomes questionable. The study of electric-field-induced surface flashover and dielectric breakdown in the LiF thin film is undertaken in this section.

It should be mentioned at the beginning that there is no experimental evidence for any ion production prior to the rise in the signals in the magnetic spectrometer, Faraday cups, the movie camera, and the ion B-dot sensors. As discussed in Section 2, these signals are interpreted to be due to the $\mathrm{Li}^{+}$ions. In addition, the earliest emission line seen is the Starkshifted Li resonance line. Early-time flashover of any significant magnitude should produce excited and ionized fluorine, as well as excited and ionized contaminant species. Such ions were observed from a LiF-coated epoxy flashover anode on the NEREUS accelerator [28]. This anode was fabricated with 0.45 - $\mathrm{mm}$-wide and 2-mm-deep grooves in an alumi- 
num surface. The width of aluminum between the grooves was $0.3 \mathrm{~mm}$. The grooves were filled with epoxy and the whole was then coated with $4 \mu \mathrm{m}$ of $\mathrm{LiF}$. The ions of the beam consisted primarily of carbon, and hydrogen, with about $20 \%$ each of lithium and fluorine. No ion production of this nature is seen on PBFA II prior to the pulse of $\mathrm{Li}^{+}$ions. A second feature to be expected from surface flashover on $\mathrm{LiF}$ is the production of intense fluorine line radiation. This was observed by Anderson during flashover on a $\mathrm{LiF}$ single crystal subjected to a surface electric field from a Febetron voltage pulse [29]. One might expect to be able to see such emission on PBFA II on shots where the optical line of sight parallel to the anode surface actually intersected the surface partially. However, no fluorine lines are seen early in time.

Although experimental data supporting a flashover mechanism on PBFA II during the time of $\mathrm{Li}^{+}$emission are clearly lacking, it still seems appropriate to try to understand the absence of flashover from a theoretical point of view. It will be implicitly assumed in what follows that there is a window of time, of the order of $10 \mathrm{~ns}$, during which dielectric breakdown of the LiF layer is most probable. That time period would commence with the arrival of the high electric fields and persist until the LiF comes under sufficient electron bombardment to be rendered conductive at the $(\Omega \mathrm{cm})^{-1}$ level as discussed in Section 4.4.

\subsection{Theoretical discussion of flashover and dielectric breakdown on a LiF anode in PBFA II}

Flashover and dielectric breakdown depend on electronic carrier multiplication in an electric field. Therefore it is necessary to begin with a discussion of the electric fields at the anode in PBFA II. In addition, the electron motion in PBFA II takes place in a very strong magnetic field, whose effects must also be taken into account. Since PBFA II is a pulsed power machine, the fields and currents are time dependent and inductive effects are important. Sections 5.2.1 and 5.2.2 develop estimates of the fields and the related parameters needed for the discussion of flashover in Sections 5.2.3, 5.2.4, and 5.2.5. Section 5.2.6 concerns the important question of how much stored electric field energy is available for $\mathrm{Li}$ desorption. The conclusion is given in Section 5.3.

\subsubsection{Principal fields, Larmor radius, effective frequency, and magnetic induction}

As discussed in Sections 2.1 and $2.2, \mathrm{Li}^{+}$ion emission occurs in PBFA II close to the time in the power pulse when the diode "saturates", i.e. when the electrons circulating in the anode-cathode gap extend from the virtual cathode to the anode [13]. Analytic theory and numerical simulations of the diode show that at this time the axial-component (vertical) of the magnetic field has reached a value near $10 \mathrm{~T}$. Its initial stationary value before the arrival of the power pulse is close to $4 \mathrm{~T}$. This increase takes place in roughly $10 \mathrm{~ns}$, from which the main angular Fourier component, $\omega$, is about $10^{8}$ radians/s. The rate of change of magnetic field, $\partial B / \partial t(B \mathrm{dot})$, is equivalent to the amplitude of $\omega \Delta B, 6 \times 10^{8} \mathrm{~T} / \mathrm{s}$. The time dependence of the principal (radial) electric field prior to the emission of $\mathrm{Li}^{+}$ions is not measured and is poorly known, but once the ions and their associated excited neutrals 
Flashover and dielectric breakdown on a

appear, the Stark shift measurements described in Section 2.1 suggest that the radial electric field at the anode is about $10 \mathrm{MV} / \mathrm{cm}$. Accordingly, the Larmor radius of an electron in these fields is approximately $60 \mu \mathrm{m}$.

\subsubsection{Tangential electric fields}

The central region of the diode is cylindrical, with an anode radius of $15.5 \mathrm{~cm}$ and a height of $20 \mathrm{~cm}$. The geometrical anode-cathode gap is typically close to $1.5 \mathrm{~cm}$. Thus it is a good approximation to treat the cylindrical diode in a one-dimensional approximation. For the purposes of this section, we will disregard the finite length of the cylinder and presume that it is much greater than the cylinder radius. Of course, this does not properly account for fringing from the ends, but the error will not be large.

Tangential electric fields at the anode surface arise from the penetration of time varying magnetic fields into the surface of the conductive anode substrate. We consider first the azimuthal electric field generated by B dot. The skin depth is very small compared to the cylinder radius, and the problem is essentially one-dimensional. The appropriate Maxwell's equations are: $\nabla \times H=J$ and $\nabla \times E=-\partial B / \partial t$. The displacement-rate term has been dropped from the first equation since the ratio of distance scale $(\ll 1 \mathrm{~cm})$ and time scale (of the order of $10 \mathrm{~ns}$ ) is much smaller than the speed of light. We know that deep within the anode conductor both $\mathbf{H}$ and $\mathbf{E}$ vanish. From this and the one-dimensional symmetry, the above vector equations can be rewritten as spatial differentials with respect to $x$, the position below the surface of the anode substrate, as follows:

$$
\begin{aligned}
& \frac{\partial B}{\partial x}=-\mu J \\
& \frac{\partial E}{\partial x}=-\frac{\partial B}{\partial t}
\end{aligned}
$$

where a consistent set of signs has been chosen to remove the vector notation and $B / \mu$ has been substituted for $H$. The current in the anode substrate is related to the induced electric field according to

$$
J=\frac{E}{\rho_{s}}
$$

where $\rho_{s}$ is the anode resistivity. If the magnetic field at the surface of the anode substrate is sinusoidally varying with amplitude $B_{o}$, the solution of (26) through (28) is

$$
B(x, t)=B_{o} \exp \left(\frac{-x}{\delta_{d}}\right) \sin \left(\omega t-\frac{x}{\delta_{d}}\right)
$$

where the skin depth, $\delta_{d}$, is given by $\left(2 p_{s} / \mu \omega\right)^{1 / 2}$. Appropriate values for the stainless steel anode are $\rho_{s}=72 \times 10^{-8} \Omega \mathrm{m}[30]$ and $\mu=4 \pi \times 10^{-7} \mathrm{Tm} / \mathrm{A}$. At $\omega=10^{8} \mathrm{sec}^{-1}$, the skin depth 
is about $100 \mu \mathrm{m}$. From (26), (28), and (29) the induced tangential electric field at the surface of the anode substrate has an amplitude of $\sqrt{2} B_{o} \rho_{s} /\left(\mu \delta_{d}\right)=B_{o}\left(\omega \rho_{s} / \mu\right)^{1 / 2}$. With $B_{o}$ set equal to the change in magnetic field, $6 \mathrm{~T}$, the induced electric field is found to be $454 \mathrm{~V} / \mathrm{cm}$, which is very small relative to the $10 \mathrm{MV} / \mathrm{cm}$ normal component.

Due to the changing magnetic field, the tangential electric field will vary with height above the anode substrate surface in the manner indicated by (27). The change in field is the product of the height and B dot. At a distance of $5 \times 10^{-5} \mathrm{~cm}$, the approximate thickness of the $\mathrm{LiF}$ layer, this field increment is about $3 \mathrm{~V} / \mathrm{cm}$ and is entirely negligible.

An axially directed (vertical) electric field is also present. The current flow from the magnetically insulated transmission line (MTL) into the anode causes an axial field component which is oppositely directed in the upper and lower halves of the anode. Maxima of this current are found at the ends of the anode, where the current is about $10^{6} \mathrm{~A}$, as discussed in Section 2.1. The maximum sheet current is therefore $10^{6}$ A divided by the anode circumference, or about $10^{6} \mathrm{~A} / \mathrm{m}$. By comparison the azimuthal component of sheet current has an amplitude of $B_{o} / \mu=4.8 \times 10^{6} \mathrm{~A} / \mathrm{m}$, according to the integrated form of (26), or about 5 times greater than the axial component. As a rough approximation, the axial current is presumed to rise according to the angular frequency, $\omega$, which characterizes the principal $\mathrm{B}$ dot of the diode. The skin depths of the two current components are then identical, and the maximum axially-directed electric field component is therefore about 5 times smaller than the azimuthal component. The vector sum of these two orthogonal fields components is of the order of $500 \mathrm{~V} / \mathrm{cm}$, or more than four orders of magnitude smaller than the normal electric field component. The electric field vector at the anode therefore deviates from normal incidence by less than 0.01 degree.

It has already been demonstrated that magnetic induction causes a negligible change in the tangential electric field at a height corresponding to the thickness of the LiF layer. It might seem possible, however, that the deposition of free charge on the LiF surface (perhaps accompanying the development of a hypothesized surface flashover) could substantially modify the tangential electric field, perhaps increasing it to a value comparable with the $10 \mathrm{MV} / \mathrm{cm}$ normal component. But this would be impossible over a lateral distance much larger than the LiF film thickness without invoking an unphysically large normal electric field component inside the $\mathrm{LiF}$ layer. Clearly, because of the extremely large ratio of the anode lateral dimensions and LiF film thickness $\left(>10^{5}\right)$, the tangential electric field on the LiF surface is closely constrained to track the tangential field established by the substrate.

\subsubsection{Flashover across the LiF surface}

The near-normal incidence of the electric field prevents secondary electron avalanches along the LiF surface. Emitted electrons follow trajectories that return them to the surface with energies nearly identical to their emission energies and no multiplication can occur. A secondary electron avalanche would not be expected unless the transverse electric field component were at least as large as the normal component [31]. 
Perhaps, if a flashover were somehow initiated, it could distort the local electric field to allow its propagation across the LiF surface. This possibility was partially addressed at the end of Section 5.2.2, but will be essentially ruled out according to Section 5.2.5.

Can the large magnetic field alter the trajectories of emitted electrons so that multiplication becomes possible? The ratio of magnetic and electric forces acting on a non-relativistic electron is given by $v_{e} B / E$, where $v_{e}$ is the electron velocity. A typical secondary electron is emitted with a kinetic energy of $10 \mathrm{eV}$ or less. The velocity associated with $10 \mathrm{eV}$ is roughly $2 \times 10^{6} \mathrm{~m} / \mathrm{s}$, and the ratio of magnetic and electric forces, using $E$ and $B$ values from Section 5.2.1, is 0.02. An equivalent finding is that the distance an electron (emitted with $10 \mathrm{eV}$ kinetic energy) can travel against the diode electric field, $10^{-6} \mathrm{~cm}$, is a very small fraction of a Larmor radius, $6 \times 10^{-3} \mathrm{~cm}$. We conclude that the magnetic field imposes no perturbation of significance on the trajectories of low energy secondary electrons.

Finally, one might suppose that early in the pulse a magnetically induced tangential electric field is briefly present before the large normal component arises, so the electric field angle at the anode would permit a secondary electron avalanche. However, the $500 \mathrm{~V} /$ $\mathrm{cm}$ tangential field from Section 5.2.2 is nearly two orders of magnitude smaller than typical fields at which surface flashover is observed to occur on a 10 ns timescale [31]. We conclude that tangential surface flashovers of any variety are highly unlikely and that the secondary electron avalanche mechanism, in particular, is not possible with the field components that are present.

\subsubsection{Flashover between LiF columns}

The columnar structure of the LiF layer is closely aligned with the diode electric field and this might allow an efficient multiplication of electrons that ricochet down the narrow voids between the crystallites. However, the total potential drop is too small to favor a runaway condition. The normal component of electric field inside the $L i F$ layer is reduced from the $10 \mathrm{MV} / \mathrm{cm}$ value in the vacuum by the effective dielectric constant, $\kappa_{e}$, of the layer. For solid $\mathrm{LiF}, \kappa_{e}=9.27$; considering the voids between the columnar crystallites, the effective dielectric constant will be somewhat less, perhaps 6 to 8 . If $\kappa_{e}$ is 6 the internal field is $1.67 \mathrm{MV} / \mathrm{cm}$ and the total potential drop across the $5 \times 10^{-5} \mathrm{~cm}$ thickness is only $83 \mathrm{~V}$. We do not accurately know at what electron kinetic energy LiF produces a secondary electron yield greater than unity, but $83 \mathrm{eV}$ is likely to be comparable to that energy [32]. The electron multiplication gain is therefore presumed to be small. Feedback of some type, for example the emission of ions or photons which start new electrons near the top of the $\mathrm{LiF}$ layer, is required for the growth of a breakdown process. We would not expect a discharge to develop without an unphysically high yield of ions or photons to compensate for the presumably low electron-multiplication gain. Surface flashover between the LiF columns is therefore judged to be quite unlikely. Solid state carrier avalanches leading to breakdown are also quite unlikely because of the small potential drop across the $\mathrm{LiF}$ layer. According to Alig and Bloom [33], carrier multiplication typically requires a deposited energy of about 2.7 times the band gap or about $36 \mathrm{eV}$ in LiF, and only a little more than two generations of multiplication could occur within the LiF layer. 


\subsubsection{Propagation of a surface discharge}

This section examines whether or not a localized breakdown in the LiF can grow laterally. Such a breakdown is presumed to result in a region of plasma, at approximately anode potential, on top of the LiF layer.

Two dimensional numerical modeling of the electric fields near the edge of a conductive layer at anode potential, on top of the $\mathrm{LiF}$, reveal a strong tangential component, as shown by the equipotentials in Figure 12. However, this field component exceeds the normal component only over a very short distance. With an effective dielectric constant $\kappa_{e}$, of 6 , the width of the region of favorable electric field components is less than $1 \%$ of the $\mathrm{LiF}$ layer thickness and spans a potential difference of less than $10 \mathrm{~V}$. Clearly, a secondary electron avalanche is not to be expected under these circumstances. Furthermore, the electric field inside the LiF is significantly enhanced only over a distance of a few percent of the $\mathrm{LiF}$ thickness and involves a potential drop of less than $30 \mathrm{~V}$. As discussed in the preceeding section, such a small potential drop makes solid-state carrier avalanches highly unlikely.

The above considerations demonstrate the extreme improbability that a localized breakdown will propagate across the LiF. One might expect, rather, that localized high fields above some critical level of a few MV/cm would rapidly decay by induced transient conduction, as observed in the work of Hibma and Zeller [34].

It is important to note that these arguments do not rule out surface flashover on conventional ion sources which have dielectric thicknesses much greater than the $\mathrm{LiF}$ thickness in PBFA II. The potential differences across regions of favorable electric field components are proportionately increased.

\subsubsection{Stored energy available for Li desorption}

Whether or not an electric discharge occurs, there is relatively little stored energy which can be converted into lithium desorption compared to the energy available in a conventional flashover source. The stored energy which can be released by electrical breakdown of the dielectric layer on the anode is the electrostatic energy contained by that layer. A complete collapse of the internal electric field would liberate a volumetric energy density of $\varepsilon_{o} E^{2} /\left(2 \kappa_{e}\right)$, which could either heat the dielectric if deposited uniformly throughout the volume, or be converted into ion or neutral desorption if deposited mainly on interfaces between the dielectric and vacuum.

In the former case, only a very small temperature rise would be expected; if the effective dielectric constant of the $\mathrm{LiF}$ layer is 6 , the stored energy density is $0.74 \mathrm{~J} / \mathrm{cm}^{3}$. Were this energy converted to thermal energy throughout the LiF volume, the temperature would rise only a fraction of a degree and no appreciable desorption would result. The same conclusion could be drawn for the dielectric material in a conventional flashover ion source operated at a corresponding internal field. 

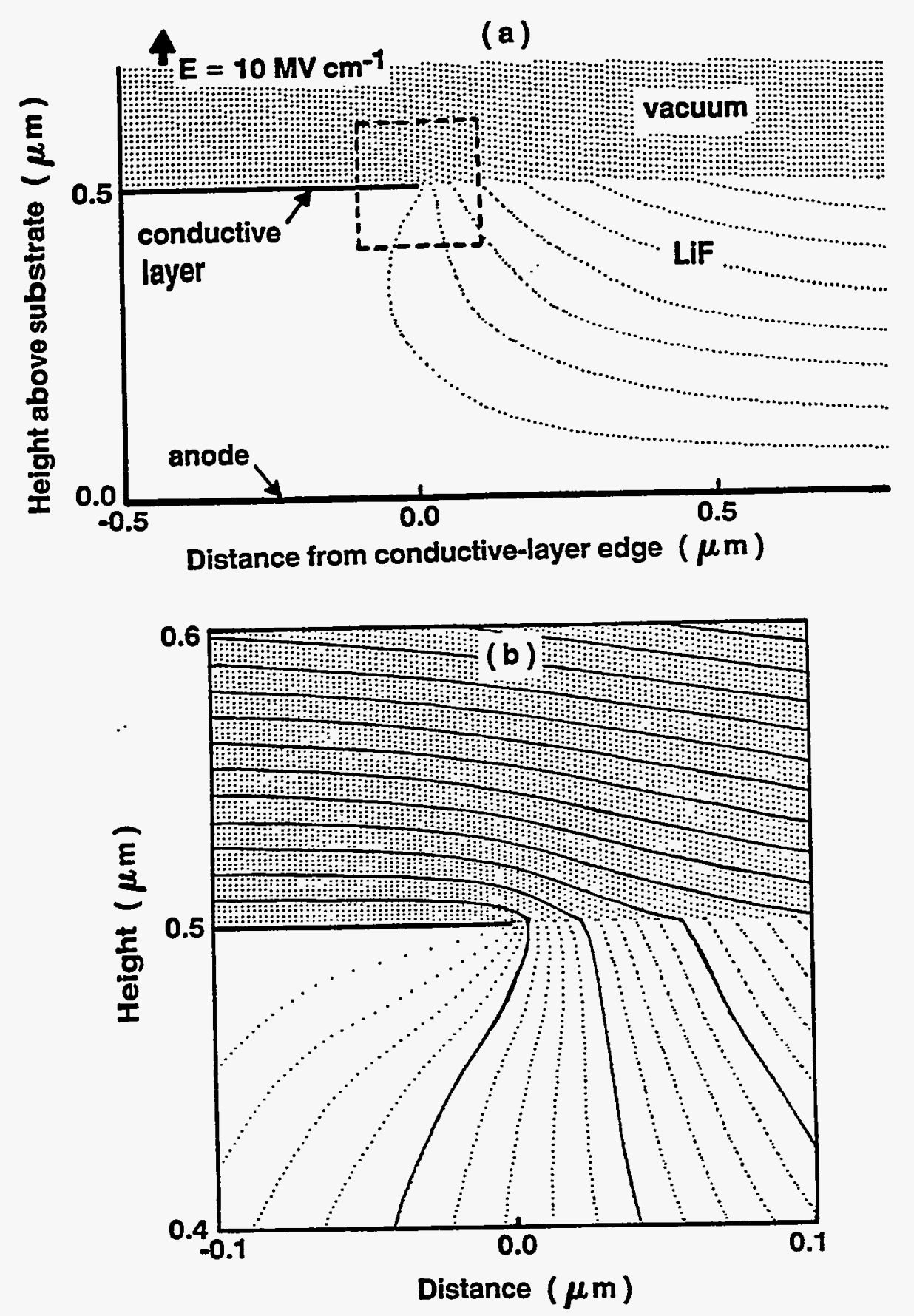

Figure 12. Calculated equipotentials in 2 dimensional case near the edge of a conductive layer (at anode potential) on top of the LiF layer, showing: a) $10 \mathrm{~V}$ equipotential lines, b) enlargement of boxed region of a), with dotted $2 \mathrm{~V}$ equipotentials and darkened 10 V equipotentials.

The crucial difference between conventional flashover ion sources and the LiF layer is the thickness of the dielectric layer. Conventional sources have dielectric inclusions with thickness of the order of millimeters; the LiF layer is more than 3 orders of magnitude thinner. If some fixed fraction of the stored energy were deposited at the free surface of the 
dielectric, as may happen with a surface flashover discharge, the areal density of this energy would be directly proportional to the layer thickness, other conditions being the same. Accordingly, the areal energy density with the LiF anode (treating the LiF surface as planar and disregarding its columnar structure) is about 3 orders of magnitude less than with conventional flashover ion sources. Under the reasonable assumption that the amount of ions or neutrals desorbed is proportional to the amount of energy deposited at the insulator surface, the $\mathrm{LiF}$ anode would be a weaker source by a factor of $10^{3}$ than a conventional flashover source.

It is tempting to think that a small fraction of the substantial electromagnetic energy stored in the anode-cathode gap might be available for conversion at the LiF surface. However, even an abrupt collapse of the internal field in the LiF layer would cause no immediate change of any consequence in either the electric or magnetic field in the gap. Therefore, until the $\mathrm{LiF}$ layer comes under bombardment from leakage electrons or irradiation by photons, the only available energy is the very small amount stored within the layer.

\subsection{Conclusion}

Based on the considerations in Section 5.2, and in particular the severely reduced energy stored in the dielectric layer by comparison with conventional flashover ion sources, we conclude with near certainty that the LiF anode in PBFA II cannot function as a flashover source. The lack of experimental evidence for $\mathrm{Li}^{+}$production by a flashover mechanism is therefore understandable.

\section{Summary}

The production of positive lithium ions using a lithium-fluoride-coated stainless steel anode in the particle beam fusion accelerator PBFA II is considered from both the experimental and theoretical points of view. It is concluded that the mechanism of $\mathrm{Li}^{+}$ion production is electric field desorption from the micron-scale crystallites which compose the columnar growth of the $\mathrm{LiF}$ thin film. The required electric field is on the order of $5 \mathrm{MV} /$ $\mathrm{cm}$. An essential feature of the mechanism is that the crystallites are rendered electronically conducting through electron-hole pair generation by $\mathrm{MeV}$ electron bombardment of the thin film during the operation of the diode. It is proposed that the ion emission mechanism is an electronic conductivity analogue to that discovered by Rollgen for lithium halide crystallites which were rendered ionically conducting by heating to several hundred degrees Centigrade.

Since an electric field desorption mechanism cannot operate if a surface flashover plasma has formed and reduced the anode electric field to low values, the possibility of flashover on the lithium-fluoride-coated anode of the PBFA II Li ${ }^{+}$ion source is studied theoretically. It is concluded with near certainty that flashover does not occur. 


\section{References}

1. R. A. Gerber, in The Physics And Technology Of Ion Sources, edited by Ian G. Brown, (Wiley, New York, 1989), Chapter 18.

2. P. F. McKay, R. A. Gerber, and A. L. Pregenzer, IEEE Transactions on Plasma Science, Vol. PS-15, No. 4, 339 (1987).

3. P. F. McKay, K. W. Bieg, R. E. Olson, A. L. Pregenzer, and D. K. Wiemann, Rev. Sci. Instrum. 61, 559 (1990).

4. A. L. Pregenzer, K. W. Bieg, R. E. Olson, and J. A. Panitz, J. Appl. Physics 67, 7556 (1990).

5. K. W. Bieg, A. L. Pregenzer, J. R. Woodworth, T. R. Lockner, D. J. Johnson, R. A. Gerber, J. E. Bailey, R. P. Kensek, R. J. Leeper, J. E. Maenchen, T. A. Mehlhorn, R. E. Olson, C. L. Ruiz, and W. A. Stygar, Rev. Sci. Instrum. 61, 556 (1990).

6. R. A. Gerber, K. W. Bieg, P. L. Dreike, P. F. McKay, A. L. Pregenzer, G. C. Tisone, and J. R. Woodworth, Rev. Sci. Instrum. 61, 511 (1990).

7. F. Okuyama, S. S. Wong, and F. W. Rollgen, Surf. Sci. 151, L131 (1985).

8. F. W. Rollgen and H.R. Schulten, Z. Naturforsch. 30, 1685 (1975).

9. F. W. Rollgen, U. Giessmann, and H. J. Heinen, Z. Naturforsch. 31a, 1729 (1976).

10. N. Itoh, Advances Phys. 31, 491 (1982).

11. J. P. VanDevender and D. L. Cook, Science 232, 831 (1986).

12. See, for example, J. E. Bailey, A. B. Filuk, A. L. Carlson, D. J. Johnson, P. Lake, E. J. McGuire, T. A. Mehlhorn, T. D. Pointon, T. J. Renk, and W. A. Stygar, and Y. Maron, Phys. Rev. Lett. 74, 1771 (1995) and references therein.

13. M. P. Desjarlais, Phys. Fluids B 1, 1709 (1989).

14. See, for example, R. J. Leeper, W. A. Stygar, J. Maenchen, C. L. Ruiz, R. P. Kensek, J. Bailey, G. Cooper, D. J. Johnson, J. R. Lee, T. R. Lockner, T. A. Mehlhorn, L. P. Mix, and R. W. Stinnett, Nucl. Instr. Methods B40/41, 973 (1989).

15. D. B. Seidel, M. L. Kiefer, R. S. Coats, T. D. Pointon, J. P. Quintenz, and W. A. Johnson, in Computational Physics, edited by A. Tenner (World Scientific, Singapore, 1991), pp 475-482.

16. M. P. Desjarlais, T. D. Pointon, D. B. Seidel, R. S. Coats, M. L. Kiefer, J. P. Quintenz, and S. A. Slutz, Phys. Rev. Lett. 67, 3094 (1991).

17. T. D. Pointon, M. P. Desjarlais, D. B. Seidel, S. A. Slutz, R. S. Coats, M. L. Kiefer, and J. P. Quintenz, Phys. Plasmas 1, 429 (1994).

18. P. R. Schwoebel and J. A. Panitz, J. Appl. Phys. 7l, 2151 (1992).

19. J. Estel, H. Hoinkes, H. Kaarmann, H. Nahr, and H. Wilsch, Surf. Sci. 54, 393 (1976).

20. P. B. Barraclough and P. G. Hall, Surf. Sci. 46, 393 (1974). 
Production of Lithium Positive Ions from LiF Thin Films on the Anode in PBFA II

21. An excellent recent book on radiation induced defects is: R. T. Williams and K. S. Song, Excitons, (Springer, 1993).

22. L. J. Lorence, Jr., J. E. Morel, and G. D. Valdez, Sandia Report, SAND89-1685 : UC505, Physics Guide to CEPXS: A Multigroup Coupled Electron-Photon Cross-Section Generating Code.

23. R. T. Williams, J. N. Bradford, and W. L. Faust, Phys. Rev. B 18, 7038 (1978).

24. C. H. Seager and D. Emin, Phys. Rev. B 2, 3421 (1960).

25. K. L. Kliewer and J. S. Koehler, Phys. Rev. 140, A1226 (1965).

26. A. E. Hughes and A. B. Lidiard, AERE R 13319, Theoretical Physics Division, Harwell Laboratory, Oxfordshire OX11 ORA, England.

27. Conference Proceedings of the XIVth International Symposium on Discharges and Electrical Insulation in Vacuum, Santa Fe, New Mexico, USA, September 17-20, 1990.

28. E. J. T. Burns, J. R. Woodworth, K. W. Bieg, T. A. Mehlhorn, W. A. Stygar, and M. A. Sweeney, J. Appl. Phys. 63, 11 (1988).

29. R. A. Anderson (unpublished).

30. CRC Handbook of Chemistry and Physics, 72nd Edition, Ed. David R. Lide, (CRC Press, Boston, 1991) p. 12-136.

31. R. A. Anderson and J. P. Brainard, J. Appl. Phys. 51, 1414 (1980).

32. I. M. Bronshteyn and A. N. Protsenko, Radio Engineering and Electronic Physics 15, 677 (1970).

33. R. C. Alig and S. Bloom, Phys. Rev. Letters 35, 1522 (1975).

34. T. Hibma and H. R. Zeller, J. Appl. Phys. 59, 1614 (1986). 


\section{DISTRIBUTION:}

5 T. A. Green

8101 Northridge, NE

Albuquerque, NM 87109

1 R. A. Gerber

3300 Rhode Island, NE

Albuquerque, NM 87110

1 SRI International

Attn: P. R. Schwoebel

333 Ravenswood Ave.

Menlo Park, CA 94025

1 Mission Research Corporation

Attn: D. Welch

1720 Randolph Road, SE

Albuquerque, NM 87106-4245

1 Laboratory of Plasma Studies

Attn: J. B. Greenly

Grumman Hall, Cornell University

Ithaca, NY 14853

1 Dept. of Physics \& Astronomy

Attn: J. A. Panitz

University of New Mexico

Albuquerque, NM 87131

3 Kernforschungszentrum Karlsruhe

Attn: H. Bluhm

Postfach 3640

Karlsruhe 1, D-76021

GERMANY

1 Weizmann Institute of Science

Attn: Y. Maron

Rehovot, Israel 76100

ISRAEL

1 MS $1413 \quad$ D. R. Jennison, 1114

$11413 \quad$ N.D. Shinn, 1114

$11423 \quad$ M. E. Riley, 1128

$11421 \quad$ G. Samara, 1152
11195 J. P. Quintenz, 1202

$1 \quad 1181 \quad$ K. R. Prestwich, 1203

$1 \quad 1182 \quad$ R. W. Stinnett, 1205

11187 T. A. Mehlhorn, 1207

$11193 \quad$ M. E. Cuneo, 1231

$1 \quad 1193 \quad$ D. Hanson, 1231

11193 D. J. Johnson, $1231^{\circ}$

$11193 \quad$ P. Menge, 1231

11193 T. J. Renk, 1231

$11186 \quad$ M. P. Desjarlais, 1241

$11186 \quad$ S. A. Slutz, 1241

11152 T. D. Pointon, 1242

$10 \quad 1187 \quad$ A. B. Filuk, 1271

11187 E. J. McGuire, 1271

11196 J. E. Bailey, 1277

$11196 \quad$ R. J. Leeper, 1277

$11196 \quad$ T. R. Lockner, 1277

$5 \quad 0367 \quad$ R. A. Anderson, 1812

$11380 \quad$ K. W. Bieg, 4202

11168 P.F.McKay, 9321

$11153 \quad$ G. M. Loubriel, 9323

$11179 \quad$ L. J. Lorence, Jr., 9341

11179 J. A. Halbleib, 9341

$10857 \quad$ M. F. Lopez, 14466

19018 Central Technical Files, 8523-2

50899 Technical Library, 13414

$10619 \quad$ Print Media, 12615

20100 Document Processing, 7613-2. For DOE/OSTI 